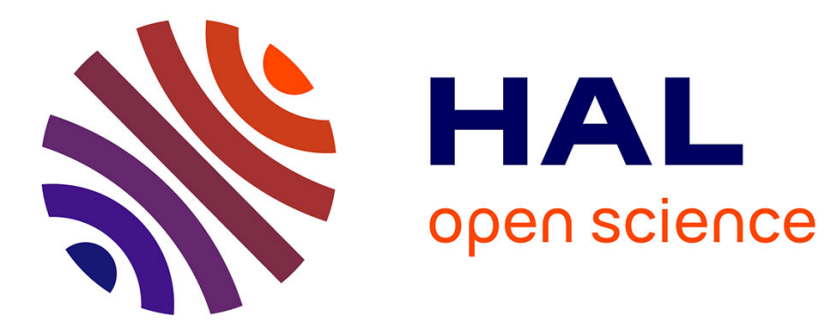

\title{
A mixing effect induced by sources concentrated in a soft junction and the gradient concentration phenomenon
} Anne-Laure Bessoud, Pongpol Juntharee, Christian Licht, Gérard Michaille

\section{To cite this version:}

Anne-Laure Bessoud, Pongpol Juntharee, Christian Licht, Gérard Michaille. A mixing effect induced by sources concentrated in a soft junction and the gradient concentration phenomenon. Asymptotic Analysis, 2012, 79, pp.303-323. 10.3233/ASY-2012-1102 . hal-00727192

\section{HAL Id: hal-00727192 \\ https://hal.science/hal-00727192}

Submitted on 3 Sep 2012

HAL is a multi-disciplinary open access archive for the deposit and dissemination of scientific research documents, whether they are published or not. The documents may come from teaching and research institutions in France or abroad, or from public or private research centers.
L'archive ouverte pluridisciplinaire HAL, est destinée au dépôt et à la diffusion de documents scientifiques de niveau recherche, publiés ou non, émanant des établissements d'enseignement et de recherche français ou étrangers, des laboratoires publics ou privés. 


\title{
A mixing effect induced by sources concentrated in a soft junction and the gradient concentration phenomenon
}

\author{
Anne-Laure Bessoud ${ }^{*}$, Pongpol Juntharee ${ }^{\dagger \ddagger}$, Christian Licht ${ }^{\dagger}$, and Gérard Michaille ${ }^{\ddagger}$
}

\begin{abstract}
We show that the variational limit of a $\varepsilon$-soft and thin junction problem $\left(\mathcal{P}_{\varepsilon}\right)$ with sources concentrated in the junction gives rise to a surface energy mixing the internal energy and sources. The surface energy functional possesses an integral representation with respect to the Gradient YoungConcentration measures generated by sequences $\left(\bar{u}_{\varepsilon}\right)_{\varepsilon>0}$ of minimizers of $\left(\mathcal{P}_{\varepsilon}\right)$.
\end{abstract}

AMS subject classifications: 35B40, 49Q20, 28A33

Keywords: $\Gamma$-convergence, Gradient Young measures, concentration measures, minimization problems.

*IMATH, EA 2134, Université du Sud Toulon-Var, Bâtiment U, BP 20132 - 83957 La Garde Cedex

${ }^{\dagger}$ LMGC, UMR-CNRS 5508, Université Montpellier II, Case courier 048, Place Eugène Bataillon, 34095 Montpellier Cedex 5

¥I3M, UMR-CNRS 5149, Université Montpellier II, Case courier 051, Place Eugène Bataillon, 34095 Montpellier Cedex 5, and MIPA, Université de Nîmes 


\section{Introduction}

This paper concerns a soft thin junction subjected to concentrated sources. More precisely, let $\Omega$ be a domain in $\mathbb{R}^{N}$ and let $B_{\varepsilon}:=\Sigma \times\left(-\frac{\varepsilon}{2}, \frac{\varepsilon}{2}\right) \subset \Omega, \Sigma \subset \mathbb{R}^{N-1}$, be the layer occupied by the soft thin junction (cf Figure 1). We consider the minimization problem

$$
\min _{u \in W_{\Gamma_{0}}^{1,2}(\Omega)}\left\{\int_{\Omega \backslash B_{\varepsilon}} f(\nabla u) d x+\varepsilon \int_{B_{\varepsilon}} g(\nabla u) d x-\left\langle\mathcal{S}^{\varepsilon}, u\right\rangle_{\varepsilon}\right\}
$$

where $W_{\Gamma_{0}}^{1,2}(\Omega)$ denotes the space of Sobolev functions with null trace on a part $\Gamma_{0}$ of the boundary of $\Omega$, and the linear form $\left\langle\mathcal{S}^{\varepsilon}, .\right\rangle_{\varepsilon}$ represents the work of the source (or the loading). Let $B:=\Sigma \times$ $\left(-\frac{1}{2}, \frac{1}{2}\right)$. A suitably rescaled $\mathcal{S}_{\varepsilon}$ of $\mathcal{S}^{\varepsilon}$ is assumed to strongly converge to some $\mathcal{S}$ in the dual of the space

$V(B):=\left\{u \in L^{2}(\Omega): \frac{\partial u}{\partial x_{N}} \in L^{2}(\Omega)\right\}$ when $\varepsilon$ tends to zero. A general example of such sources which are measures on $B_{\varepsilon}$ is given in Section 4 of the paper. Sources of the form $c \frac{1}{L(\varepsilon)} \mathbb{1}_{B_{\varepsilon}}$ where $c$ is any constant and $L(\varepsilon) \sim \varepsilon$, is a trivial example of measures satisfying this condition with $\mathcal{S}=\mathbb{1}_{B}$. Note that in this paper the source (or the loading) $\mathcal{S}_{\varepsilon}$ is a non $L^{2}$-continuous perturbation of the energy functional $\int_{\Omega \backslash B_{\varepsilon}} f(\nabla u) d x+\varepsilon \int_{B_{\varepsilon}} g(\nabla u) d x$.

Among the physical motivations of $\left(\mathcal{P}_{\varepsilon}\right)$ one may mention various applications to heat conduction or electrostatic problems involving sources concentrated in the layer $B_{\varepsilon}$ with conductivity or permittivity of order the size of $B_{\varepsilon}$. One may also think of membrane problems with an exterior loading concentrated in $B_{\varepsilon}$ occupied by a material with stiffness of order the small size of $B_{\varepsilon}$. Such a problem with a source concentrated in the junction was considered in [3] in a one dimensional case in order to highlight and illustrate a gradient concentration phenomenon, but the authors were not able to express the variational limit problem.

This paper illustrates the same gradient concentration phenomenon with a complete description of the limit problem in the sense of $\Gamma$-convergence (Theorem 3.3). When the size $\varepsilon$ of the layer goes to zero, fields $u_{\varepsilon}$ of bounded energy develop a discontinuity through $\Sigma$. More precisely, at the variational limit, the internal energy functional of the junction $\varepsilon \int_{B_{\varepsilon}} g(\nabla u) d x$ and the work of the loading $\left\langle\mathcal{S}^{\varepsilon}, u\right\rangle_{\varepsilon}$ are combined into a functional of the type

$$
\bar{H}(u)=\int_{\Sigma} \bar{h}\left(\hat{x}, u^{+}-u^{-}, \frac{u^{+}+u^{-}}{2}\right) d \hat{x}
$$

and the limit problem reads as

$$
\min _{u \in W_{\Gamma_{0}}^{1,2}(\Omega \backslash \Sigma)}\left\{\int_{\Omega} f(\nabla u) d x+\bar{H}(u)\right\}
$$

where $u^{ \pm}$denote the traces on $\Sigma$. When regarding the various studies devoted to the asymptotic modeling of junction problems (see $[2,9,7,10]$ and references therein) the main novelty is that the density $\bar{h}$ depends also of the mean $\frac{u^{+}+u^{-}}{2}$. Furthermore we show that the sequence of minimizers of $\left(\mathcal{P}_{\varepsilon}\right)$ (which converges to a minimizer $\bar{u}$ of the limit problem $(\mathcal{P})$ ) generates a gradient Young-concentration measure $\bar{\mu}$ in the sense defined in [3]. Then we can give an integral representation of the internal part of $\bar{H}$ with respect to the measure $\bar{\mu}$ (Theorem 5.5) so that it can be localized in $\Sigma \times\{ \pm 1\}$. Finally this provides new bounds on the measure $\bar{\mu}$ (Corollary 5.6).

The paper is organized as follows: in Section 2 we fix notation and give a detailed description of the problem $\left(\mathcal{P}_{\varepsilon}\right)$. Section 3 is devoted to the asymptotic analysis of $\left(\mathcal{P}_{\varepsilon}\right)$ in the sense of the $\Gamma$-convergence of the functional energy extended to $L^{2}(\Omega)$ equipped with its strong topology. In Section 4 we describe a large class of suitable sources $\mathcal{S}_{\varepsilon}$. Finally Section 5 is concerned with the analysis of the gradient concentration phenomenon generated by sequences of minimizers of $\left(\mathcal{P}_{\varepsilon}\right)$. We stress the fact that one could treat the problem in $L^{p}(\Omega), 1<p<+\infty$ in the same way without additional difficulties. 


\section{Description of the minimization problem}

Let $\varepsilon>0$ be a small parameter intended to go to zero, more precisely taking values in a countable subset of $\left(0, \varepsilon_{0}\right]$ whose 0 is the only cluster point. The reference configuration of the assembly of the two adherents and the adhesive is a cylinder $\Omega:=\Sigma \times(-r, r)$ (with $r>\varepsilon$ ), where $\Sigma$ is a bounded domain in $\mathbb{R}^{N-1}, N \geq 2$, with Lipschitz boundary. For $x \in \mathbb{R}^{N}$ we sometimes write $x=\left(\hat{x}, x_{N}\right)$ where $\hat{x} \in \mathbb{R}^{N-1}$. In all the paper, $C$ denotes a non negative constant which does not depend on $\varepsilon$ and may vary from line to line. We do not relabel the various considered subsequences and the symbols $\rightarrow$ and $\rightarrow$ denote various strong convergences and weak convergences respectively. We define the following sets:

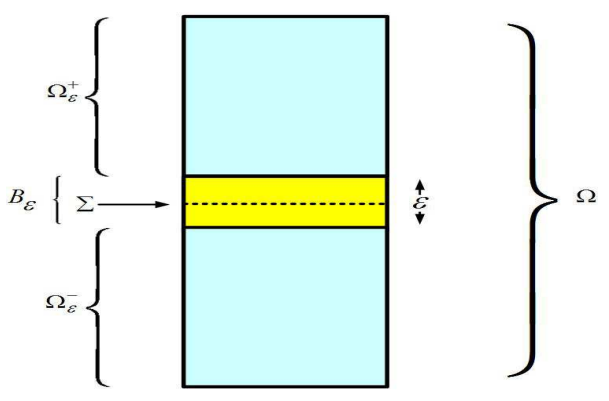

Figure 1: physical domain

. $B_{\varepsilon}:=\Sigma \times\left(-\frac{\varepsilon}{2}, \frac{\varepsilon}{2}\right)$;

. $B:=\Sigma \times\left(-\frac{1}{2}, \frac{1}{2}\right)$;

. $\Omega_{\varepsilon}=\Omega \backslash \overline{B_{\varepsilon}}$

. $\Gamma_{0}$ is a subset of the boundary $\partial \Omega$ of $\Omega$ such that $\operatorname{dist}\left(\bar{\Gamma}_{0}, \overline{\partial B_{\varepsilon} \cap \partial \Omega}\right)>0$ for all $\varepsilon<\varepsilon_{0}$;

. we write $\Omega_{\varepsilon}^{-}, \Omega_{\varepsilon}^{+}, \Omega^{-}, \Omega^{+}, B_{\varepsilon}^{+}$and $B_{\varepsilon}^{-}$for the sets $\Omega_{\varepsilon} \cap\left[x_{N}<0\right]$ and $\Omega_{\varepsilon} \cap\left[x_{N}>0\right], \Omega \cap\left[x_{N}<0\right]$, $\Omega \cap\left[x_{N}>0\right]$ and $B_{\varepsilon} \cap\left[x_{N}>0\right], B_{\varepsilon} \cap\left[x_{N}<0\right]$ respectively.

We will be concerned with the following spaces:

. $W_{\Gamma_{0}}^{1,2}\left(\Omega_{\varepsilon}\right):=\left\{u \in W^{1,2}\left(\Omega_{\varepsilon}\right): u=0\right.$ on $\left.\Gamma_{0}\right\} ;$

. $W_{\Gamma_{0}}^{1,2}(\Omega):=\left\{u \in W^{1,2}(\Omega): u=0\right.$ on $\left.\Gamma_{0}\right\}$;

. $W_{\Gamma_{0}}^{1,2}(\Omega \backslash \Sigma):=\left\{u \in W^{1,2}(\Omega \backslash \Sigma): u=0\right.$ on $\left.\Gamma_{0}\right\}$, and for every $z \in W_{\Gamma_{0}}^{1,2}(\Omega \backslash \Sigma), z^{ \pm}$will stand for the traces of $z$ on $\Sigma$ considered as a Sobolev function on $\Omega^{+}$and $\Omega^{-}$respectively.

We say that a function $h: \mathbb{R}^{N} \longrightarrow \mathbb{R} \cup\{+\infty\}$ satisfies a growth condition of order 2 if there exist $\alpha$ and $\beta$ in $\mathbb{R}^{+}$such that

$$
\alpha|\xi|^{2} \leq h(\xi) \leq \beta\left(1+|\xi|^{2}\right) \text { for all } \xi \in \mathbb{R}^{N} .
$$

We consider two convex functions $f, g: \mathbb{R}^{N} \longrightarrow \mathbb{R}$ satisfying a growth condition of order 2 , and we assume that there exists a positively 2-homogeneous function $g^{\infty, 2}$ satisfying

$$
\left|g(\xi)-g^{\infty, 2}(\xi)\right| \leq \beta\left(1+|\xi|^{2-\delta}\right) \text { for all } \xi \in \mathbb{R}^{N},
$$


for some $\delta, 0<\delta<2$. Note that $g^{\infty, 2}$ is the positively 2-homogeneous recession function of $g$, i.e.,

$$
g^{\infty, 2}(\xi)=\lim _{t \rightarrow+\infty} \frac{g(t \xi)}{t^{2}}
$$

is convex and satisfies the same growth condition of order 2 . We define the space

$$
V\left(B_{\varepsilon}\right):=\left\{u \in L^{2}\left(B_{\varepsilon}\right): \frac{\partial u}{\partial x_{N}} \in L^{2}\left(B_{\varepsilon}\right)\right\}
$$

equipped with the norm

$$
\|u\|_{V\left(B_{\varepsilon}\right)}:=\left(\int_{B_{\varepsilon}}|u|^{2} d x+\int_{B_{\varepsilon}}\left|\frac{\partial u}{\partial x_{N}}\right|^{2} d x\right)^{\frac{1}{2}}
$$

and we denote the duality bracket between the topological dual space $V^{\prime}\left(B_{\varepsilon}\right)$ and $V\left(B_{\varepsilon}\right)$ by $\langle,\rangle_{\varepsilon}$. The considered total energy functional $F_{\varepsilon}: L^{2}(\Omega) \longrightarrow \mathbb{R} \cup\{+\infty\}$ is defined by

$$
F_{\varepsilon}(u)=\left\{\begin{array}{l}
\int_{\Omega_{\varepsilon}} f(\nabla u) d x+\varepsilon \int_{B_{\varepsilon}} g(\nabla u) d x-\left\langle\mathcal{S}^{\varepsilon}, u\right\rangle_{\varepsilon} \text { if } u \in W_{\Gamma_{0}}^{1,2}(\Omega) \\
+\infty \text { otherwise }
\end{array}\right.
$$

where $\mathcal{S}^{\varepsilon}$ is given in $V^{\prime}\left(B_{\varepsilon}\right)$. Our aim is to describe the asymptotic behavior of the minimization problem

$$
\left(\mathcal{P}_{\varepsilon}\right) \quad \min \left\{F_{\varepsilon}(u): u \in L^{2}(\Omega)\right\},
$$

namely, the limit of $\min \left\{F_{\varepsilon}(u): u \in L^{2}(\Omega)\right\}$ together with the limit of the minimizer $\bar{u}_{\varepsilon}$, and to identify the limit problem in the framework of $\Gamma$-convergence.

Let us consider the space $V(B):=\left\{u \in L^{2}(B): \frac{\partial u}{\partial x_{N}} \in L^{2}(B)\right\}$ equipped with the norm

$$
\|u\|_{V(B)}:=\left(\int_{B}|u|^{2} d x+\int_{B}\left|\frac{\partial u}{\partial x_{N}}\right|^{2} d x\right)^{\frac{1}{2}},
$$

and denote the duality bracket between $V^{\prime}(B)$ and $V(B)$ by $\langle.,$.$\rangle . The linear continuous operator$

$$
\tau_{\varepsilon}: V\left(B_{\varepsilon}\right) \longrightarrow V(B)
$$

is defined for every $x=\left(\hat{x}, x_{N}\right) \in B$ by $\tau_{\varepsilon} u\left(\hat{x}, x_{N}\right):=u\left(\hat{x}, \varepsilon x_{N}\right)$ and we denote its transposed operator by ${ }^{T} \tau_{\varepsilon}$ :

$$
\left\langle{ }^{T} \tau_{\varepsilon} \theta, u\right\rangle_{\varepsilon}=\left\langle\theta, \tau_{\varepsilon} u\right\rangle, \forall(\theta, u) \in V^{\prime}(B) \times V\left(B_{\varepsilon}\right) .
$$

We make the following assumption on the source $\mathcal{S}^{\varepsilon}$ : there exists $\mathcal{S}$ in $V^{\prime}(B)$ such that

$$
S_{\varepsilon}:=\left({ }^{T} \tau_{\varepsilon}\right)^{-1} \mathcal{S}^{\varepsilon} \text { strongly converges to } \mathcal{S} \text { in } V^{\prime}(B) .
$$

Then, in order to identify the $\Gamma$-limit of the functional $F_{\varepsilon}$, it will be more convenient to write the functional $F_{\varepsilon}$ as

$$
F_{\varepsilon}(u)=\left\{\begin{array}{l}
\int_{\Omega_{\varepsilon}} f(\nabla u) d x+\varepsilon^{2} \int_{B} g\left(\widehat{\nabla} \tau_{\varepsilon} u, \frac{1}{\varepsilon} \frac{\partial \tau_{\varepsilon} u}{\partial x_{N}}\right) d x-\left\langle\mathcal{S}_{\varepsilon}, \tau_{\varepsilon} u\right\rangle \text { if } u \in W_{\Gamma_{0}}^{1,2}(\Omega) \\
+\infty \text { otherwise. }
\end{array}\right.
$$

\section{The variational asymptotic model}

Let $H: V(B) \longrightarrow \mathbb{R}$ be the functional defined by

$$
\begin{aligned}
H(\theta) & :=\int_{B} g^{\infty, 2}\left(\hat{0}, \frac{\partial \theta}{\partial x_{N}}\right) d x-\langle\mathcal{S}, \theta\rangle \\
& =H_{i n}(\theta)-\langle\mathcal{S}, \theta\rangle .
\end{aligned}
$$


We refer the fiunctional $H_{i n}$ as the internal part of $H$. We claim that, when $L^{2}(\Omega)$ is equipped with its strong topology, the functional $F_{\varepsilon} \Gamma$-converges to the functional $F_{0}: L^{2}(\Omega) \longrightarrow \mathbb{R} \cup\{+\infty\}$ given by

$$
F_{0}(u)=\left\{\begin{array}{l}
\int_{\Omega} f(\nabla u) d x+\inf _{\theta \in X(u)} H(\theta) \text { if } u \in W_{\Gamma_{0}}^{1,2}(\Omega \backslash \Sigma), \\
+\infty \text { otherwise, }
\end{array}\right.
$$

where $X(u):=\left\{\theta \in V(B): \theta\left(., \pm \frac{1}{2}\right)=u^{ \pm}\right\}$.

Before addressing the variational convergence process, we begin by establishing some compactness properties for sequences with bounded energy. Let us introduce the $\varepsilon$-translate operator $T_{\varepsilon}$ from $W^{1,2}(\Omega)$ into $W^{1,2}(\Omega \backslash \Sigma)$. For any function $w \in W^{1,2}(\Omega), \tilde{w}$ stands for its extension by reflexion on $\Sigma \times(-2 r,-r) \cup$ $(r, 2 r)$ and we define the $\varepsilon$-translate $T_{\varepsilon} w$ of $w$ by

$$
T_{\varepsilon} w\left(\hat{x}, x_{N}\right)= \begin{cases}\widetilde{w}\left(\hat{x}, x_{N}+\frac{\varepsilon}{2}\right) & \text { if } x \in \Omega^{+} \\ \widetilde{w}\left(\hat{x}, x_{N}-\frac{\varepsilon}{2}\right) & \text { if } x \in \Omega^{-}\end{cases}
$$

Lemma 3.1 (compactness). Let $\left(u_{\varepsilon}\right)_{\varepsilon>0}$ be a sequence in $L^{2}(\Omega)$ such that $\sup _{\varepsilon>0} F_{\varepsilon}\left(u_{\varepsilon}\right)<+\infty$. Then

$$
\int_{B_{\varepsilon}}\left|u_{\varepsilon}\right|^{2} d x \leq C \varepsilon\left(\int_{\Omega_{\varepsilon}}\left|\nabla u_{\varepsilon}\right|^{2} d x+\varepsilon \int_{B_{\varepsilon}}\left|\frac{\partial u_{\varepsilon}}{\partial x_{N}}\right|^{2} d x\right)
$$

(ii)

$$
\sup _{\varepsilon>0}\left(\int_{\Omega_{\varepsilon}}\left|\nabla u_{\varepsilon}\right|^{2} d x+\varepsilon \int_{B_{\varepsilon}}\left|\frac{\partial u_{\varepsilon}}{\partial x_{N}}\right|^{2} d x\right)<+\infty
$$

(iii) there exist $u \in W_{\Gamma_{0}}^{1,2}(\Omega \backslash \Sigma)$ and a subsequence of $\left(u_{\varepsilon}\right)_{\varepsilon>0}$ such that $u_{\varepsilon} \rightarrow u$ in $L^{2}(\Omega)$ and $u_{\varepsilon} \rightarrow u$ in $W_{\Gamma_{0}}^{1,2}\left(\Omega_{\eta}\right)$ for all $\eta>0$;

(iv) there exist $\theta \in V(B)$ and a subsequence such that $\tau_{\varepsilon} u_{\varepsilon} \rightarrow \theta$ in $V(B)$, i.e.

$$
\begin{aligned}
& \tau_{\varepsilon} u_{\varepsilon} \rightarrow \theta \text { in } L^{2}(B), \\
& \frac{\partial \tau_{\varepsilon} u_{\varepsilon}}{\partial x_{N}} \rightarrow \frac{\partial \theta}{\partial x_{N}} \text { in } L^{2}(B) ;
\end{aligned}
$$

moreover, $\varepsilon \hat{\nabla} \tau_{\varepsilon} u_{\varepsilon} \rightarrow 0$ in $L^{2}\left(B, \mathbb{R}^{N-1}\right) ;$

(v) $\theta\left(., \pm \frac{1}{2}\right)=u^{ \pm}$.

Proof. Proof of (i). Without loss of generality, we may assume that the $N$-1-dimensional Hausdorff measure of the intersection of $\Gamma_{0}$ with $\left[x_{N}>0\right]$ is positive so that (i) is a mere consequence of the following Poincaré-like inequality:

$$
\exists C>0, \int_{B_{\varepsilon}}|\varphi|^{2} d x \leq C \varepsilon\left(\int_{\Omega_{\varepsilon}^{+}}|\nabla \varphi|^{2} d x+\varepsilon \int_{B_{\varepsilon}}\left|\frac{\partial \varphi}{\partial x_{N}}\right|^{2} d x\right) \forall \varphi \in W_{\Gamma_{0}}^{1,2}(\Omega) .
$$

Indeed, because

$$
\varphi\left(\hat{x}, x_{N}\right)=T_{\varepsilon} \varphi(\hat{x}, 0)+\int_{\frac{\varepsilon}{2}}^{x_{N}} \frac{\partial}{\partial x_{N}} \varphi(\hat{x}, t) d t \quad \forall x \in B_{\varepsilon},
$$

for all smooth function $\varphi \in W_{\Gamma_{0}}^{1,2}(\Omega)$, we get

$$
\left|\varphi\left(\hat{x}, x_{N}\right)\right|^{2} \leq 2\left(\left|T_{\varepsilon} \varphi(\hat{x}, 0)\right|^{2}+\varepsilon \int_{-\frac{\varepsilon}{2}}^{\frac{\varepsilon}{2}}\left|\frac{\partial}{\partial x_{N}} \varphi(\hat{x}, t)\right|^{2} d t\right) .
$$

Hence, integrating on $B_{\varepsilon}$ and using trace inequality and Poincaré inequality in $\Omega^{+}$give the desired inequality (3.4) for smooth $\varphi$, thus for all $\varphi$ in $W_{\Gamma_{0}}^{1,2}(\Omega)$ by a density argument. 
Proof of (ii). From the coercivity conditions satisfied by $f$ and $g$, estimate (3.2), and the strong convergence of $\mathcal{S}_{\varepsilon}$ in $V^{\prime}(B)$, one has

$$
\begin{aligned}
\alpha\left(\int_{\Omega_{\varepsilon}}\left|\nabla u_{\varepsilon}\right|^{2} d x+\varepsilon \int_{B_{\varepsilon}}\left|\frac{\partial u_{\varepsilon}}{\partial x_{N}}\right|^{2} d x\right) & \leq C+\left|\left\langle\mathcal{S}^{\varepsilon}, u_{\varepsilon}\right\rangle_{\varepsilon}\right| \\
& =C+\left|\left\langle\mathcal{S}_{\varepsilon}, \tau_{\varepsilon} u_{\varepsilon}\right\rangle\right| \\
& \leq C+\left\|\mathcal{S}_{\varepsilon}\right\|_{V^{\prime}(B)}\left\|\tau_{\varepsilon} u_{\varepsilon}\right\|_{V(B)} \\
& =C+\left\|\mathcal{S}_{\varepsilon}\right\|_{V^{\prime}(B)}\left(\frac{1}{\varepsilon} \int_{B_{\varepsilon}}\left|u_{\varepsilon}\right|^{2} d x+\varepsilon \int_{B_{\varepsilon}}\left|\frac{\partial u_{\varepsilon}}{\partial x_{N}}\right|^{2} d x\right)^{\frac{1}{2}} \\
& \leq C+C\left(\int_{\Omega_{\varepsilon}}\left|\nabla u_{\varepsilon}\right|^{2} d x+\varepsilon \int_{B_{\varepsilon}}\left|\frac{\partial u_{\varepsilon}}{\partial x_{N}}\right|^{2} d x\right)^{1 / 2} .
\end{aligned}
$$

Then, setting $X_{\varepsilon}:=\left(\int_{\Omega_{\varepsilon}}\left|\nabla u_{\varepsilon}\right|^{2} d x+\varepsilon \int_{B_{\varepsilon}}\left|\frac{\partial u_{\varepsilon}}{\partial x_{N}}\right|^{2} d x\right)^{1 / 2}$, (3.3) follows from the estimate $\alpha X_{\varepsilon}^{2} \leq$ $C+C X_{\varepsilon}$.

Proof of (iii).

Step 1. We claim that there exist $z \in W^{1,2}(\Omega \backslash \Sigma)$ and a subsequence of $\left(u_{\varepsilon}\right)_{\varepsilon>0}$ such that $T_{\varepsilon} u_{\varepsilon} \rightarrow z$ in $W^{1,2}(\Omega \backslash \Sigma)$ and strongly in $L^{2}(\Omega \backslash \Sigma)$. Clearly,

$$
T_{\varepsilon} u_{\varepsilon} \in W^{1,2}(\Omega \backslash \Sigma) \text { and } \frac{\partial}{\partial x_{i}} T_{\varepsilon} u_{\varepsilon}=T_{\varepsilon} \frac{\partial}{\partial x_{i}} u_{\varepsilon} \text { for all } \varepsilon>0 .
$$

Combining the Poincaré inequality, (3.3) and (3.5), we deduce

$$
\sup _{\varepsilon>0}\left\|T_{\varepsilon} u_{\varepsilon}\right\|_{W^{1,2}(\Omega \backslash \Sigma)}^{2} \leq C \sup _{\varepsilon>0}\left(\int_{\Omega_{\varepsilon}}\left|\nabla u_{\varepsilon}(x)\right|^{2} d x+\varepsilon \int_{B_{\varepsilon}}\left|\frac{\partial u_{\varepsilon}}{\partial x_{N}}(x)\right|^{2} d x\right)<+\infty .
$$

Therefore, $\left(T_{\varepsilon} u_{\varepsilon}\right)_{\varepsilon>0}$ is bounded in $W^{1,2}(\Omega \backslash \Sigma)$ and the claim follows immediately.

Step 2. We establish that there exists $u$ in $L^{2}(\Omega)$ such that we can extract from the previous subsequence $\left(u_{\varepsilon}\right)_{\varepsilon>0}$ a subsequence strongly converging to $u$ in $L^{2}(\Omega)$. We can write

$$
\int_{\Omega_{\varepsilon}}\left|u_{\varepsilon}(x)\right|^{2} d x=\int_{\Omega^{+} \cup \Omega^{-}}\left|T_{\varepsilon} u_{\varepsilon}(x)\right|^{2} d x-\int_{\Sigma \times\left(\left(r-\frac{\varepsilon}{2}, r\right) \cup\left(-r,-r+\frac{\varepsilon}{2}\right)\right)}\left|T_{\varepsilon} u_{\varepsilon}(x)\right|^{2} d x
$$

so that

$$
\left\|u_{\varepsilon}\right\|_{L^{2}(\Omega)}^{2}=\int_{\Omega^{+} \cup \Omega^{-}}\left|T_{\varepsilon} u_{\varepsilon}(x)\right|^{2} d x+\int_{B_{\varepsilon}}\left|u_{\varepsilon}(x)\right|^{2} d x-\int_{\Sigma \times\left(\left(r-\frac{\varepsilon}{2}, r\right) \cup\left(-r,-r+\frac{\varepsilon}{2}\right)\right)}\left|T_{\varepsilon} u_{\varepsilon}(x)\right|^{2} d x .
$$

From step 1 and (3.2), we deduce that $\sup _{\varepsilon>0}\left\|u_{\varepsilon}\right\|_{L^{2}(\Omega)}<+\infty$. Thus there exist $u \in L^{2}(\Omega)$ and a not relabelled subsequence such that $u_{\varepsilon} \rightarrow u$ in $L^{2}(\Omega)$. Let us prove that $u=z$. Since $u_{\varepsilon} \rightarrow u$ in $L^{2}(\Omega)$ and $T_{\varepsilon} u_{\varepsilon} \rightarrow z$ in $W^{1,2}(\Omega \backslash \Sigma)$, we have for any $\varphi \in C_{c}^{\infty}(\Omega)$,

$$
\begin{aligned}
\int_{\Omega} u(x) \varphi(x) d x & =\lim _{\varepsilon \rightarrow 0} \int_{\Omega} u_{\varepsilon}(x) \varphi\left(\widehat{x}, x_{N}-\frac{\varepsilon}{2}\right) d x \\
& =\lim _{\varepsilon \rightarrow 0} \int_{\Omega} T_{\varepsilon} u_{\varepsilon}(x) \varphi(x) d x \\
& =\int_{\Omega} z(x) \varphi(x) d x .
\end{aligned}
$$

Thus $u=z$ almost everywhere in $\Omega$ and we deduce that $u \in W^{1,2}(\Omega \backslash \Sigma)$.

Moreover, from (3.2) we have that $\int_{B_{\varepsilon}}\left|u_{\varepsilon}(x)\right|^{2} d x \rightarrow 0$ as $\varepsilon \rightarrow 0$. On the other hand, since $T_{\varepsilon} u_{\varepsilon} \rightarrow z$ 
in $L^{2}(\Omega)$, we infer $\int_{\Omega^{+} \cup \Omega^{-}}\left|T_{\varepsilon} u_{\varepsilon}(x)\right|^{2} d x \rightarrow \int_{\Omega}|z(x)|^{2} d x$ and $\int_{\Sigma \times\left(\left(r-\frac{\varepsilon}{2}, r\right) \cup\left(-r,-r+\frac{\varepsilon}{2}\right)\right)}\left|T_{\varepsilon} u_{\varepsilon}(x)\right|^{2} d x \rightarrow 0$. Then we deduce that $\left\|u_{\varepsilon}\right\|_{L^{2}(\Omega)} \rightarrow\|z\|_{L^{2}(\Omega)}=\|u\|_{L^{2}(\Omega)}$ and thus that $\left(u_{\varepsilon}\right)_{\varepsilon>0}$ strongly converges to $u$ in $L^{2}(\Omega)$.

Step 3. It remains to establish that for any $\eta>0$, there exists a subsequence of $\left(u_{\varepsilon}\right)_{\varepsilon>0}$ such that $u_{\varepsilon\left\lfloor\Omega_{\eta}\right.} \rightarrow u_{\left\lfloor\Omega_{\eta}\right.}$ in $W_{\Gamma_{0}}^{1,2}\left(\Omega_{\eta}\right)$. It will immediately result that $u \in W_{\Gamma_{0}}^{1,2}(\Omega \backslash \Sigma)$.

Let $\eta>0$. Clearly, there exists $0<\varepsilon_{1}<\eta$ such that $\Omega_{\eta} \subseteq \Omega_{\varepsilon}$ for all $\varepsilon \leq \varepsilon_{1}$. By the Poincaré inequality we have

$$
\sup _{\varepsilon>0}\left\|u_{\varepsilon}\right\|_{W^{1,2}\left(\Omega_{\eta}\right)}^{2} \leq C \sup _{\varepsilon>0}\left(\int_{\Omega_{\varepsilon}}\left|\nabla u_{\varepsilon}(x)\right|^{2} d x+\varepsilon \int_{B_{\varepsilon}}\left|\frac{\partial u_{\varepsilon}}{\partial x_{N}}(x)\right|^{2} d x\right)<+\infty .
$$

Thus, $\left(u_{\varepsilon}\right)_{\varepsilon>0}$ is bounded in $W_{\Gamma_{0}}^{1,2}\left(\Omega_{\eta}\right)$, and there exist $w \in W_{\Gamma_{0}}^{1,2}\left(\Omega_{\eta}\right)$ and a not relabelled subsequence of $\left(u_{\varepsilon}\right)_{\varepsilon>0}$ satisfying $u_{\varepsilon} \rightarrow w$ in $L^{2}\left(\Omega_{\eta}\right)$ and $u_{\varepsilon} \rightarrow w$ in $W_{\Gamma_{0}}^{1,2}\left(\Omega_{\eta}\right)$. It is easily seen that in fact $w=u_{\left\lfloor\Omega_{\eta}\right.}$.

Proof of (iv). The weak convergence of $\tau_{\varepsilon} u_{\varepsilon}$ to some $\theta$ in $V(B)$ follows from (3.2) and (3.3). Indeed

$$
\begin{aligned}
\sup _{\varepsilon>0}\left\|\tau_{\varepsilon} u_{\varepsilon}\right\|_{V(B)} & =\sup _{\varepsilon>0}\left(\frac{1}{\varepsilon} \int_{B_{\varepsilon}}\left|u_{\varepsilon}\right|^{2} d x+\varepsilon \int_{B_{\varepsilon}}\left|\frac{\partial u_{\varepsilon}}{\partial x_{N}}\right|^{2} d x\right)^{\frac{1}{2}} \\
& \leq C \sup _{\varepsilon>0} X_{\varepsilon}<+\infty .
\end{aligned}
$$

Now we deduce that $\widehat{\nabla} \tau_{\varepsilon} u_{\varepsilon} \rightarrow \widehat{\nabla} \theta$ in the distributional sense so that $\varepsilon \hat{\nabla} \tau_{\varepsilon} u_{\varepsilon} \rightarrow 0$ in the distributional sense. On the other hand, from the coercivity of $g, \varepsilon \widehat{\nabla} \tau_{\varepsilon} u_{\varepsilon}$ weakly converges to some $L^{2}\left(B, \mathbb{R}^{N-1}\right)$ function. Hence, $\varepsilon \hat{\nabla} \tau_{\varepsilon} u_{\varepsilon} \rightarrow 0$ in $L^{2}\left(B, \mathbb{R}^{N-1}\right)$.

Proof of (v). Note that $\theta\left(., \pm \frac{1}{2}\right)$ is well defined. Indeed, one has

$$
V(B) \subset W^{1,2}\left(\left(-\frac{1}{2}, \frac{1}{2}\right), L^{2}(\Sigma)\right) \subset \mathcal{C}\left(\left[-\frac{1}{2}, \frac{1}{2}\right], L^{2}(\Sigma)\right) .
$$

Clearly, $\tau_{\varepsilon} u_{\varepsilon}\left(\hat{x}, \pm \frac{1}{2}\right)=\left(T_{\varepsilon} u_{\varepsilon}\right)^{ \pm}(\hat{x})$ (in the sense of traces on $\Sigma$ of $W_{\Gamma_{0}}^{1,2}(\Omega \backslash \Sigma)$-functions) so that $\tau_{\varepsilon} u_{\varepsilon}\left(\hat{x}, \pm \frac{1}{2}\right) \rightarrow u^{ \pm}$in $L^{2}(\Sigma)$. On the other hand, since

$$
\tau_{\varepsilon} u_{\varepsilon}\left(\hat{x}, x_{N}\right)=\tau_{\varepsilon} u_{\varepsilon}\left(\hat{x}, \pm \frac{1}{2}\right)+\int_{ \pm \frac{1}{2}}^{x_{N}} \frac{\partial \tau_{\varepsilon} u_{\varepsilon}}{\partial x_{N}}(\hat{x}, s) d s
$$

for a.e. $x$ in $B$, we infer that for all $\varphi \in \mathcal{C}_{c}(\Sigma)$,

$$
\int_{-\frac{1}{2}}^{\frac{1}{2}} \int_{\Sigma} \tau_{\varepsilon} u_{\varepsilon}\left(\hat{x}, x_{N}\right) \varphi(\hat{x}) d x=\int_{\Sigma}\left(T_{\varepsilon} u_{\varepsilon}\right)^{ \pm}(\hat{x}) \varphi(\hat{x}) d \hat{x}+\int_{-\frac{1}{2}}^{\frac{1}{2}} \int_{\Sigma} \int_{ \pm \frac{1}{2}}^{x_{N}} \frac{\partial \tau_{\varepsilon} u_{\varepsilon}}{\partial x_{N}}(\hat{x}, s) \varphi(\hat{x}) d s d x .
$$

By passing to the limit in (3.7), we obtain

$$
\int_{-\frac{1}{2}}^{\frac{1}{2}} \int_{\Sigma} \theta\left(\hat{x}, x_{N}\right) \varphi(\hat{x}) d x=\int_{\Sigma} u^{ \pm}(\hat{x}) \varphi(\hat{x}) d \hat{x}+\int_{-\frac{1}{2}}^{\frac{1}{2}} \int_{\Sigma} \int_{ \pm \frac{1}{2}}^{x_{N}} \frac{\partial \theta}{\partial x_{N}}(\hat{x}, s) \varphi(\hat{x}) d s d x
$$

from which we deduce

$$
\int_{\Sigma} u^{ \pm}(\hat{x}) \varphi(\hat{x}) d \hat{x}=\int_{\Sigma} \theta\left(\hat{x}, \pm \frac{1}{2}\right) \varphi(\hat{x}) d \hat{x} .
$$

Thus $\theta\left(., \pm \frac{1}{2}\right)=u^{ \pm}$almost everywhere in $\Sigma$.

Lemma 3.2. For every $u \in W_{\Gamma_{0}}^{1,2}(\Omega \backslash \Sigma), \inf _{\theta \in X(u)} H(\theta)>-\infty$ and there exists $\theta(u) \in X(u)$ such that $\inf _{\theta \in X(u)} H(\theta)=H(\theta(u))$. 
Proof. The proof follows from standard arguments used in the direct method of the Calculus of Variation.

As a consequence of Lemma 3.2, in its domain $W_{\Gamma_{0}}^{1,2}(\Omega \backslash \Sigma)$, the functional $F_{0}$ may be written

$$
F_{0}(u)=\int_{\Omega} f(\nabla u) d x+H(\theta(u)) .
$$

Theorem 3.3 is the main result of this section.

Theorem 3.3. The sequence $\left(F_{\varepsilon}\right)_{\varepsilon>0} \Gamma$-converges to the functional $F_{0}$ when $L^{2}(\Omega)$ is equipped with its strong topology.

The proof results from the following two propositions.

Proposition 3.4. For every $u \in L^{2}(\Omega)$ and every $\left(u_{\varepsilon}\right)_{\varepsilon>0}$ strongly converging to $u$ in $L^{2}(\Omega)$ one has

$$
F_{0}(u) \leq \liminf _{\varepsilon \rightarrow 0} F_{\varepsilon}\left(u_{\varepsilon}\right) .
$$

Proposition 3.5. For every $u \in L^{2}(\Omega)$ there exists $\left(v_{\varepsilon}\right)_{\varepsilon>0}$ strongly converging to $u$ in $L^{2}(\Omega)$ satisfying

$$
F_{0}(u) \geq \limsup _{\varepsilon \rightarrow 0} F_{\varepsilon}\left(v_{\varepsilon}\right) .
$$

Proof of Proposition 3.4. We may assume $\liminf _{\varepsilon \rightarrow 0} F_{\varepsilon}\left(u_{\varepsilon}\right)<+\infty$. From Lemma $3.1 u \in W_{\Gamma_{0}}^{1,2}(\Omega \backslash \Sigma)$ and there exists $\theta \in X(u)$ such that $\tau_{\varepsilon} u_{\varepsilon} \rightarrow \theta$ in $V(B)$. Since $\mathcal{S}_{\varepsilon} \rightarrow \mathcal{S}$ in $V^{\prime}(B)$, one has

$$
\lim _{\varepsilon \rightarrow 0}\left\langle\mathcal{S}_{\varepsilon}, \tau_{\varepsilon} u_{\varepsilon}\right\rangle=\langle\mathcal{S}, \theta\rangle \text {. }
$$

On the other hand, since from Lemma 3.1, $u_{\varepsilon} \rightarrow u$ in $W_{\Gamma_{0}}^{1,2}\left(\Omega_{\eta}\right)$ for all $\eta>0$, one has

$$
\liminf _{\varepsilon \rightarrow 0} \int_{\Omega_{\varepsilon}} f\left(\nabla u_{\varepsilon}\right) d x \geq \int_{\Omega} f(\nabla u) d x .
$$

Finally from (iv) of Lemma 3.1 and a standard lower semicontinuity argument

$$
\begin{aligned}
& \liminf _{\varepsilon \rightarrow 0} \varepsilon^{2} \int_{B} g\left(\widehat{\nabla} \tau_{\varepsilon} u_{\varepsilon}, \frac{1}{\varepsilon} \frac{\partial\left(\tau_{\varepsilon} u_{\varepsilon}\right)}{\partial x_{N}}\right) d x \\
\geq & \liminf _{\varepsilon \rightarrow 0}\left(\varepsilon^{2} \int_{B} g\left(\widehat{\nabla} \tau_{\varepsilon} u_{\varepsilon}, \frac{1}{\varepsilon} \frac{\partial\left(\tau_{\varepsilon} u_{\varepsilon}\right)}{\partial x_{N}}\right) d x-\int_{B} g^{\infty, 2}\left(\varepsilon \widehat{\nabla} \tau_{\varepsilon} u_{\varepsilon}, \frac{\partial\left(\tau_{\varepsilon} u_{\varepsilon}\right)}{\partial x_{N}}\right) d x\right) \\
& +\liminf _{\varepsilon \rightarrow 0} \int_{B} g^{\infty, 2}\left(\varepsilon \widehat{\nabla} \tau_{\varepsilon} u_{\varepsilon}, \frac{\partial\left(\tau_{\varepsilon} u_{\varepsilon}\right)}{\partial x_{N}}\right) d x \\
\geq & \liminf _{\varepsilon \rightarrow 0}\left(\varepsilon^{2} \int_{B} g\left(\widehat{\nabla} \tau_{\varepsilon} u_{\varepsilon}, \frac{1}{\varepsilon} \frac{\partial\left(\tau_{\varepsilon} u_{\varepsilon}\right)}{\partial x_{N}}\right) d x-\int_{B} g^{\infty, 2}\left(\varepsilon \hat{\nabla} \tau_{\varepsilon} u_{\varepsilon}, \frac{\partial\left(\tau_{\varepsilon} u_{\varepsilon}\right)}{\partial x_{N}}\right) d x\right) \\
& +\int_{B} g^{\infty, 2}\left(\hat{0}, \frac{\partial \theta}{\partial x_{N}}\right) d x \\
= & \int_{B} g^{\infty, 2}\left(\hat{0}, \frac{\partial \theta}{\partial x_{N}}\right) d x
\end{aligned}
$$

provided that we establish

$$
\lim _{\varepsilon \rightarrow 0}\left(\varepsilon^{2} \int_{B} g\left(\widehat{\nabla} \tau_{\varepsilon} u_{\varepsilon}, \frac{1}{\varepsilon} \frac{\partial\left(\tau_{\varepsilon} u_{\varepsilon}\right)}{\partial x_{N}}\right) d x-\int_{B} g^{\infty, 2}\left(\varepsilon \widehat{\nabla} \tau_{\varepsilon} u_{\varepsilon}, \frac{\partial\left(\tau_{\varepsilon} u_{\varepsilon}\right)}{\partial x_{N}}\right) d x\right)=0 .
$$

Since $g^{\infty, 2}$ is positively homogeneous of degree 2 , and from (2.1), we have

$$
\begin{aligned}
& \int_{B}\left|\varepsilon^{2} g\left(\widehat{\nabla} \tau_{\varepsilon} u_{\varepsilon}, \frac{1}{\varepsilon} \frac{\partial\left(\tau_{\varepsilon} u_{\varepsilon}\right)}{\partial x_{N}}\right)-g^{\infty, 2}\left(\varepsilon \widehat{\nabla} \tau_{\varepsilon}^{-1} u_{\varepsilon}, \frac{\partial\left(\tau_{\varepsilon} u_{\varepsilon}\right)}{\partial x_{N}}\right)\right| d x \\
= & \varepsilon^{2} \int_{B}\left|g\left(\widehat{\nabla} \tau_{\varepsilon} u_{\varepsilon}, \frac{1}{\varepsilon} \frac{\partial\left(\tau_{\varepsilon} u_{\varepsilon}\right)}{\partial x_{N}}\right)-g^{\infty, 2}\left(\widehat{\nabla} \tau_{\varepsilon} u_{\varepsilon}, \frac{1}{\varepsilon} \frac{\partial\left(\tau_{\varepsilon} u_{\varepsilon}\right)}{\partial x_{N}}\right)\right| d x \\
\leq & C \varepsilon^{2} \int_{B}\left[1+\left|\widehat{\nabla} \tau_{\varepsilon} u_{\varepsilon}\right|^{2-\delta}+\left|\frac{1}{\varepsilon} \frac{\partial\left(\tau_{\varepsilon} u_{\varepsilon}\right)}{\partial x_{N}}\right|^{2-\delta}\right] d x .
\end{aligned}
$$


Thus, by using Hölder's inequality (take $p=\frac{2}{2-\delta}, q=\frac{2}{\delta}$ ) we deduce

$$
\int_{B}\left|\varepsilon^{2} g\left(\widehat{\nabla} \tau_{\varepsilon} u_{\varepsilon}, \frac{1}{\varepsilon} \frac{\partial \tau_{\varepsilon} u_{\varepsilon}}{\partial x_{N}}\right)-g^{\infty, 2}\left(\varepsilon \widehat{\nabla} \tau_{\varepsilon} u_{\varepsilon}, \frac{\partial\left(\tau_{\varepsilon} u_{\varepsilon}\right)}{\partial x_{N}}\right)\right| d x \leq C \varepsilon^{\delta}
$$

which proves (3.11). The conclusion of Proposition 3.4 follows by collecting (3.8), (3.9) and (3.10).

Proof of Proposition 3.5. Let $u \in L^{2}(\Omega)$. We have to construct a sequence $\left(v_{\varepsilon}\right)_{\varepsilon>0}$ strongly converging to $u$ in $L^{2}(\Omega)$ such that $\limsup F_{\varepsilon}\left(v_{\varepsilon}\right) \leq F_{0}(u)$. If $F_{0}(u)=+\infty$, then $u \in L^{2}(\Omega) \backslash W_{\Gamma_{0}}^{1,2}(\Omega \backslash \Sigma)$, and clearly, for any sequence $\left(v_{\varepsilon}\right)_{\varepsilon>0}$ converging to $u, \limsup _{\varepsilon \rightarrow 0} F_{\varepsilon}\left(v_{\varepsilon}\right) \leq F_{0}(u)$ is true. Now, for the harder part, we assume $F_{0}(u)<+\infty$. Then $u \in W_{\Gamma_{0}}^{1,2}(\Omega \backslash \Sigma)$ and

$$
F_{0}(u)=\int_{\Omega} f(\nabla u(x)) d x+\inf _{\theta \in X(u)} H(\theta) .
$$

To complete the proof, from $\bar{\theta}:=\theta(u)$, i.e. $H(\bar{\theta})=\inf _{\theta \in X(u)} H(\theta)$, we construct a sequence $\left(v_{\varepsilon}\right)_{\varepsilon>0}$ strongly converging to $u$ in $L^{2}(\Omega)$ and satisfying

$$
F_{0}(u) \geq \limsup _{\varepsilon \rightarrow 0} F_{\varepsilon}\left(v_{\varepsilon}\right) .
$$

The proof is divided into four steps:

Step 1. Let us extend $u$ and $\bar{\theta}$ by 0 into $\left(\mathbb{R}^{N-1} \backslash \Sigma\right) \times(-r, r)$ and write these extensions $\widetilde{u}$ and $\widetilde{\bar{\theta}}$. For a sequence $\delta$ of positive numbers intended to go to 0 , consider a standard sequence of molifier $\left(\rho_{\delta}\right)_{\delta}$ and set

$$
\begin{array}{ll}
u_{\delta}:=\rho_{\delta} * \widetilde{u} \text { defined by } \rho_{\delta} * \widetilde{u}\left(\widehat{x}, x_{N}\right)=\int_{\mathbb{R}^{N-1}} \rho_{\delta}(\widehat{x}-\widehat{y}) \widetilde{u}\left(\widehat{y}, x_{N}\right) d \widehat{y} & \text { for all }\left(\widehat{x}, x_{N}\right) \in \Omega ; \\
\theta_{\delta}:=\rho_{\delta} * \widetilde{\bar{\theta}} \text { defined by } \rho_{\delta} * \widetilde{\bar{\theta}}\left(\widehat{x}, x_{N}\right)=\int_{\mathbb{R}^{N-1}} \rho_{\delta}(\widehat{x}-\widehat{y}) \widetilde{\bar{\theta}}\left(\widehat{y}, x_{N}\right) d \widehat{y} & \text { for all }\left(\widehat{x}, x_{N}\right) \in \Omega .
\end{array}
$$

Clearly,

$$
\left\{\begin{array}{l}
\theta_{\delta}\left(\widehat{x}, \pm \frac{1}{2}\right)=u_{\delta}(\widehat{x}, 0) \text { for all } \widehat{x} \in \Sigma, \\
u_{\delta} \in W^{1,2}(\Omega \backslash \Sigma), \theta_{\delta} \in W^{1,2}(B), \\
u_{\delta} \rightarrow u \text { in } W^{1,2}(\Omega \backslash \Sigma), \theta_{\delta} \rightarrow \bar{\theta} \text { in } V(B) .
\end{array}\right.
$$

Next, for each $\delta>0$, we define the sequence $\left(v_{\delta, \varepsilon}\right)_{\varepsilon>0}$ as follows:

$$
v_{\delta, \varepsilon}\left(\widehat{x}, x_{N}\right)=\left\{\begin{array}{lll}
u_{\delta}\left(\widehat{x}, x_{N} \pm \frac{\varepsilon}{2}\right) & \text { on } & \Omega_{\varepsilon}^{\mp} \\
\theta_{\delta}\left(\widehat{x}, \frac{x_{N}}{\varepsilon}\right) & \text { on } \quad B_{\varepsilon} .
\end{array}\right.
$$

Obviously $v_{\delta, \varepsilon}\left(\widehat{x}, x_{N}\right)$ belongs to $W^{1,2}(\Omega)$ and strongly converges to $u_{\delta}$ in $L^{2}(\Omega)$.

Step 2. We we claim that

$$
\begin{aligned}
& \lim _{\varepsilon \rightarrow 0} \int_{\Omega_{\varepsilon}} f\left(\nabla v_{\delta, \varepsilon}\right)(x) d x=\int_{\Omega} f\left(\nabla u_{\delta}\right)(x) d x \\
& \lim _{\varepsilon \rightarrow 0}\left(\varepsilon^{2} \int_{B} g\left(\widehat{\nabla} \tau_{\varepsilon} v_{\delta, \varepsilon}, \frac{1}{\varepsilon} \frac{\partial \tau_{\varepsilon} v_{\delta, \varepsilon}}{\partial x_{N}}\right)(x) d x-\left\langle\mathcal{S}_{\varepsilon}, \tau_{\varepsilon} v_{\delta, \varepsilon}\right\rangle\right)=H\left(\theta_{\delta}\right) .
\end{aligned}
$$

Proof of (3.14): one has

$$
\begin{aligned}
\lim _{\varepsilon \rightarrow 0} \int_{\Omega_{\varepsilon}} f\left(\nabla v_{\delta, \varepsilon}\right)(x) d x & =\lim _{\varepsilon \rightarrow 0}\left(\int_{\Omega_{\varepsilon}^{+}} f\left(\nabla u_{\delta}\right)\left(\widehat{x}, x_{N}-\frac{\varepsilon}{2}\right) d x+\int_{\Omega_{\varepsilon}^{-}} f\left(\nabla u_{\delta}\left(\widehat{x}, x_{N}+\frac{\varepsilon}{2}\right)\right) d x\right) \\
& =\int_{\Omega^{+}} f\left(\nabla u_{\delta}\right)(x) d x+\int_{\Omega^{-}} f\left(\nabla u_{\delta}\right)(x) d x \\
& =\int_{\Omega} f\left(\nabla u_{\delta}\right)(x) d x .
\end{aligned}
$$


Proof of (3.15): Since $g^{\infty, 2}$ is positively homogeneous of degree 2 and $\mathcal{S}_{\varepsilon}$ strongly converges to $\mathcal{S}$ in $V^{\prime}(B)$, one has

$$
\lim _{\varepsilon \rightarrow 0}\left(\varepsilon^{2} \int_{B} g\left(\widehat{\nabla} \theta_{\delta}, \frac{1}{\varepsilon} \frac{\partial \theta_{\delta}}{\partial x_{N}}\right)(x) d x-\left\langle\mathcal{S}_{\varepsilon}, \theta_{\delta}\right\rangle\right)=\int_{B} g^{\infty, 2}\left(\widehat{0}, \frac{\partial \theta_{\delta}}{\partial x_{N}}\right) d x-\left\langle\mathcal{S}, \theta_{\delta}\right\rangle=H\left(\theta_{\delta}\right) .
$$

Step 3. We establish that $\lim _{\delta \rightarrow 0} \int_{\Omega} f\left(\nabla u_{\delta}\right) d x+H\left(\theta_{\delta}\right)=F_{0}(u)$. Since

$$
\int_{\Omega} f\left(\nabla u_{\delta}\right) d x+H\left(\theta_{\delta}\right)=\int_{\Omega} f\left(\nabla u_{\delta}\right) d x+\int_{B} g^{\infty, 2}\left(\widehat{0}, \frac{\partial \theta_{\delta}}{\partial x_{N}}\right) d x-\left\langle\mathcal{S}, \theta_{\delta}\right\rangle,
$$

the result is a straightforward consequence of (3.12).

Step 4. By using a standard diagonalization argument, from step 2 and step 3, there exists a mapping $\varepsilon \mapsto \delta(\varepsilon)$ such that $v_{\delta(\varepsilon)} \longrightarrow u$ in $L^{2}(\Omega)$ and

$$
\lim _{\varepsilon \rightarrow 0}\left(\int_{\Omega} f\left(\nabla v_{\delta(\varepsilon)}\right)(x) d x+\varepsilon^{2} \int_{B} g\left(\widehat{\nabla} \tau_{\varepsilon} v_{\delta(\varepsilon)}, \frac{1}{\varepsilon} \frac{\partial \tau_{\varepsilon} v_{\delta(\varepsilon)}}{\partial x_{N}}\right)(x) d x-\left\langle\mathcal{S}_{\varepsilon}, \tau_{\varepsilon} v_{\delta(\varepsilon)}\right\rangle\right)=F_{0}(u) .
$$

The sequence $\left(v_{\varepsilon}\right)_{\varepsilon>0}$ where $v_{\varepsilon}:=v_{\delta(\varepsilon)}$ fullfils all the conditions except the boundary condition on $\Gamma_{0}$. From assumption $\operatorname{dist}\left(\bar{\Gamma}_{0}, \overline{\partial B_{\varepsilon} \cap \partial \Omega}\right)>0$, and by using a standard slicing method due to De Giorgi in a neighborhood of $\Gamma_{0}$ (see [4]), one can modify $v_{\varepsilon}$ in $\Omega_{\varepsilon}$ into a function $\tilde{v}_{\varepsilon}$ equal to $v_{\varepsilon}$ in $B_{\varepsilon}$, satisfying the boundary condition on $\Gamma_{0}$, and $\lim \sup _{\varepsilon \rightarrow 0} \int_{\Omega_{\varepsilon}} f\left(\nabla v_{\varepsilon}\right) d x=\lim \sup _{\varepsilon \rightarrow 0} \int_{\Omega_{\varepsilon}} f\left(\nabla \tilde{v}_{\varepsilon}\right) d x$. Still denoting by $v_{\varepsilon}$ this new function, we have $\lim _{\varepsilon \rightarrow 0} F_{\varepsilon}\left(v_{\varepsilon}\right)=F_{0}(u)$ and the proof is complete.

Remark 3.6. In order to give an interpretation of the limit energy functional, it is worthwhile to write

$$
\inf _{\theta \in X(u)} H(\theta)=\inf _{\theta \in V_{0}(B)}\left\{\int_{B} g^{\infty, 2}\left(\hat{0}, \frac{\partial \theta}{\partial x_{N}}(x)+[u](\hat{x})\right) d x-\langle\mathcal{S}, \theta\rangle\right\}-\langle\mathcal{S}, \tilde{u}\rangle
$$

where $[u]=u^{+}-u^{-}, V_{0}(B)=\left\{\theta \in V(B): \theta=0\right.$ on $\left.\Sigma \times\left\{ \pm \frac{1}{2}\right\}\right\}$ and $\tilde{u}(x)=x_{N}[u](\hat{x})+\frac{u^{+}(\hat{x})+u^{-}(\hat{x})}{2}$. Thererfore when the limit source $\mathcal{S}$ vanishes on $V(B)$, by using Jensen's inequality, $\inf _{\theta \in X(u)} H(\theta)$ reduces to

$$
\bar{H}(u)=\int_{\Sigma} g^{\infty, 2}(\hat{0},[u](\hat{x})) d \hat{x}
$$

which is nothing but the surface energy of the model obtained in [9]. When the limit source is not trivial, by using the Euler equation associated with (3.16), it is easily seen that $\bar{H}$ is a surface energy on $\Sigma$ of the form

$$
\bar{H}(u)=\int_{\Sigma} \bar{h}\left(\hat{x},[u](\hat{x}), \frac{u^{+}+u^{-}}{2}(\hat{x})\right) d \hat{x} .
$$

In this case we note that the energy density depends explicitly of the mean of the traces and that the surface energy $\bar{H}$ mixes the internal energy and the work of the loading.

\section{Examples of measure sources $\mathcal{S}_{\varepsilon}$ concentrated in $B_{\varepsilon}$}

The general form of elements of $V^{\prime}(B)$ is given for every $\theta$ in $V(B)$ by $\langle\mathcal{S}, \theta\rangle=\int_{B} s_{0} \theta d x+\int_{B} s_{1} \frac{\partial \theta}{\partial x_{N}} d x$ where $\left(s_{0}, s_{1}\right) \in L^{2}(B) \times L^{2}(B)$. The limit sources $\mathcal{S}$ considered in this section are generated by measures $\mathcal{S}^{\varepsilon}$ in $\mathbb{M}\left(B_{\varepsilon}\right)$ whose slicing structure $\mathcal{H}^{N-1}\left\lfloor\Sigma \otimes \mathcal{S}_{\hat{x}}^{\varepsilon}\right.$ is such that their slicing components $\mathcal{S}_{\hat{x}}^{\varepsilon}$ do not present a diffuse singular part in their Lebesgue-Nikodym decomposition in $\mathbb{M}\left(-\frac{\varepsilon}{2}, \frac{\varepsilon}{2}\right)$, i.e., are of the general form

$$
\mathcal{S}_{\hat{x}}^{\varepsilon}=\frac{1}{\varepsilon} a_{\varepsilon}(\hat{x}, \varepsilon t) d t+\sum_{n=-\infty}^{+\infty} b_{\varepsilon, n}(\hat{x}) \delta_{\varepsilon t_{n}(\hat{x})}
$$


where

$$
\left\{\begin{array}{l}
a_{\varepsilon} \in L^{2}(B), b_{\varepsilon, n} \in L^{2}(\Sigma) \\
t_{n}: \Sigma \longrightarrow\left(-\frac{1}{2}, \frac{1}{2}\right) \text { is a Borel measurable map. }
\end{array}\right.
$$

Roughly, such sources $\mathcal{S}^{\varepsilon}$ are sums of a function in $L^{2}(B)$ and a countable sum of surface sources, each of them being concentrated in the $N$-1-dimensional surface included in $B_{\varepsilon}$ whose graph is $\varepsilon t_{n}$. We make the following additional assumptions:

(H1) there exists $a \in L^{2}(B)$ such that $a_{\varepsilon} \rightarrow a$ in $L^{2}(B)$;

(H2) there exists $b_{n} \in L^{2}(\Sigma)$ such that $b_{\varepsilon, n} \rightarrow b_{n}$ in $L^{2}(\Sigma)$ when $\varepsilon \rightarrow 0$;

(H3) there exists $c_{n} \in \mathbb{R}^{+}$such that $\left\|b_{\varepsilon, n}\right\|_{L^{2}(\Sigma)} \leq c_{n}$ and $\sum_{n=-\infty}^{+\infty} c_{n}<+\infty$;

It is easy to check that the measure $\mathcal{S}_{\varepsilon}={ }^{T} \tau_{\varepsilon} \mathcal{S}^{\varepsilon}$ of $\mathbb{M}(B)$ is given by: $\mathcal{S}_{\varepsilon}=\mathcal{H}^{N-1}\left\lfloor\Sigma \otimes\left(\mathcal{S}_{\varepsilon}\right)_{\hat{x}}\right.$ where

$$
\left(\mathcal{S}_{\varepsilon}\right)_{\hat{x}}=a_{\varepsilon}(\hat{x}, t) d t+\sum_{n=-\infty}^{+\infty} b_{\varepsilon, n}(\hat{x}) \delta_{t_{n}(\hat{x})} .
$$

Proposition 4.1. The measure $\mathcal{S}_{\varepsilon}$ strongly converges in $V^{\prime}(B)$ to the measure $\mathcal{S}$ defined for every $\theta \in V(B)$ by

$$
\langle\mathcal{S}, \theta\rangle=\int_{B} a(x) \theta(x) d x+\sum_{n=-\infty}^{+\infty} \int_{\Sigma} b_{n}(\hat{x}) \theta\left(\hat{x}, t_{n}(\hat{x})\right) d \hat{x} .
$$

Therefore, the functional $F_{\varepsilon} \Gamma$-converges to the functional $F_{0}: L^{2}(\Omega) \longrightarrow \mathbb{R} \cup\{+\infty\}$ given by

$$
F_{0}(u)=\left\{\begin{array}{l}
\int_{\Omega} f(\nabla u) d x+\inf _{\theta \in X(u)}\left\{\int_{B} g^{\infty, 2}\left(\hat{0}, \frac{\partial \theta}{\partial x_{N}}\right) d x-\int_{B} a \theta d x-\sum_{n=-\infty}^{+\infty} \int_{\Sigma} b_{n}(\hat{x}) \theta\left(\hat{x}, t_{n}(\hat{x})\right) d \hat{x}\right\} \\
\text { if } u \in W_{\Gamma_{0}}^{1,2}(\Omega \backslash \Sigma)
\end{array}\right.
$$

Proof. The second assertion is a straightforward consequence of Theorem 3.3 provided that we establish the strong convergence of $\mathcal{S}_{\varepsilon}$ to $\mathcal{S}$ in $V^{\prime}(B)$. For every $\theta \in V(B)$ we have

$$
\left\langle\mathcal{S}_{\varepsilon}-\mathcal{S}, \theta\right\rangle=\int_{B}\left(a_{\varepsilon}-a\right) \theta d x+\int_{\Sigma} \sum_{n=-\infty}^{+\infty}\left(b_{\varepsilon, n}-b_{n}\right) \theta\left(\hat{x}, t_{n}(\hat{x})\right) d \hat{x}
$$

thus

$$
\left|\left\langle\mathcal{S}_{\varepsilon}-\mathcal{S}, \theta\right\rangle\right| \leq\|\theta\|_{L^{2}(B)}\left\|a_{\varepsilon}-a\right\|_{L^{2}(B)}+\sum_{n=-\infty}^{+\infty}\left[\left\|b_{\varepsilon, n}-b_{n}\right\|_{L^{2}(\Sigma)}\left(\int_{\Sigma} \mid \theta\left(\hat{x},\left.t_{n}(\hat{x})\right|^{2} d \hat{x}\right)^{\frac{1}{2}}\right] .\right.
$$

But it is easy to establish that there exists a non negative constant $C$ such that

$$
\left(\int_{\Sigma} \mid \theta\left(\hat{x},\left.t_{n}(\hat{x})\right|^{2} d \hat{x}\right)^{\frac{1}{2}} \leq C\|\theta\|_{V(B)}\right.
$$

so that (4.1) yields

$$
\left\|\mathcal{S}_{\varepsilon}-\mathcal{S}\right\|_{V^{\prime}(B)} \leq\left\|a_{\varepsilon}-a\right\|_{L^{2}(B)}+C \sum_{n=-\infty}^{+\infty}\left\|b_{\varepsilon, n}-b_{n}\right\|_{L^{2}(\Sigma)}
$$

The conclusion follows from assumptions $(H 1),(H 2)$ and $(H 3)$. 


\section{The gradient concentration phenomenon}

We first recall the notion of gradient Young-concentration measure introduced in [3]. Let us denote the unit sphere $\{-1,1\}$ of $\mathbb{R}$ by $\mathbb{S}^{0}$, and consider $\Sigma^{\prime} \subset \subset \Sigma, B_{\varepsilon}^{\prime}:=\Sigma^{\prime} \times\left(-\frac{\varepsilon}{2}, \frac{\varepsilon}{2}\right)$.

Definition 5.1. A pair $\left(v, \mu_{\Sigma^{\prime}}\right) \in L^{2}(\Omega) \times \mathbb{M}^{+}\left(\bar{\Omega} \times \mathbb{S}^{0}\right)$ is a gradient Young-concentration measure (localized on $\Sigma^{\prime}$ ) iff there exists a sequence $\left(v_{\varepsilon}\right)_{\varepsilon>0}$ in $W_{\Gamma_{0}}^{1,2}(\Omega)$ satisfying

$$
\left\{\begin{array}{l}
\sup _{\varepsilon>0} \int_{\Omega \backslash B_{\varepsilon}}\left|\nabla v_{\varepsilon}\right|^{2} d x<+\infty \\
v_{\varepsilon} \rightarrow v \text { in } L^{2}(\Omega), \\
\mu_{\varepsilon}:=\delta_{\frac{\partial v_{\varepsilon}}{\partial x_{N}} /\left|\frac{\partial v_{\varepsilon}}{\partial x_{N}}\right|(x)} \otimes \varepsilon \mathbb{1}_{B_{\varepsilon}^{\prime}}\left|\frac{\partial v_{\varepsilon}}{\partial x_{N}}\right|^{2} d x \stackrel{*}{\rightarrow} \mu_{\Sigma^{\prime}} .
\end{array}\right.
$$

We say that the sequence $\left(v_{\varepsilon}\right)_{\varepsilon>0}$ generates the gradient Young-concentration measure $\left(v, \mu_{\Sigma^{\prime}}\right)$. We denote the set of gradient Young-concentration measures localized on $\Sigma^{\prime}$ by $\mathcal{Y C}\left(\Sigma^{\prime}\right)$.

Recall that the weak convergence $\stackrel{*}{\rightarrow}$ above is defined by

$$
\int_{\bar{\Omega}} \int_{\mathbb{S}^{0}} \theta(x) \varphi(\zeta) d \mu_{\varepsilon}=\int_{B_{\varepsilon}^{\prime}} \varepsilon \theta(x) \tilde{\varphi}\left(\frac{\partial v_{\varepsilon}}{\partial x_{N}}\right) d x \rightarrow \int_{\bar{\Omega}} \int_{\mathbb{S}^{0}} \theta(x) \varphi(\zeta) d \mu_{\Sigma^{\prime}}
$$

for all $\theta \in \mathcal{C}(\bar{\Omega})$ and all $\varphi \in \mathcal{C}\left(\mathbb{S}^{0}\right)$, where the 2-homogeneous extension $\tilde{\varphi}: \mathbb{R} \rightarrow \mathbb{R}$ of $\varphi \in \mathcal{C}\left(\mathbb{S}^{0}\right)$ is defined for all $\zeta \in \mathbb{R}^{m}$ by

$$
\tilde{\varphi}(\zeta)= \begin{cases}|\zeta|^{2} \varphi\left(\frac{\zeta}{|\zeta|}\right), & \text { if } \zeta \neq 0 \\ 0 & \text { otherwise }\end{cases}
$$

In [3], Theorem 3.1, the gradient Young-concentration measures was characterized as follows.

Theorem 5.2 (Characterization). A pair $\left(v, \mu_{\Sigma^{\prime}}=\mu_{x} \otimes \pi\right)$ belongs to $\mathcal{Y C}\left(\Sigma^{\prime}\right)$ if and only if $v \in W_{\Gamma_{0}}^{1,2}(\Omega \backslash$ $\Sigma), \pi$ is concentrated on $\bar{\Sigma}^{\prime}$ and, for every $\varphi \in \mathcal{C}\left(\mathbb{S}^{0}\right)$ such that $\varphi^{* *}>-\infty$,

$$
\begin{aligned}
& \frac{d \pi}{d \mathcal{H}^{N-1}\left\lfloor\Sigma^{\prime}\right.}(x) \int_{\mathbb{S}^{0}} \varphi(\zeta) d \mu_{x} \geq \varphi^{* *}([v](x)) \quad \text { for } \mathcal{H}^{N-1} \text { a. e. } x \in \Sigma^{\prime} \\
& \int_{\mathbb{S}^{0}} \varphi(\zeta) d \mu_{x} \geq 0 \quad \text { for } \pi_{s} \text { a. e. } x \in \bar{\Sigma}^{\prime}
\end{aligned}
$$

where $\pi=\frac{d \pi}{d \mathcal{H}^{N-1}\left[\Sigma^{\prime}\right.} \mathcal{H}^{N-1}\left\lfloor\Sigma^{\prime}+\pi_{s}\right.$ is the Radon-Nikodym decomposition of $\pi$ with respect to the measure $\mathcal{H}^{N-1}\left\lfloor\Sigma^{\prime}\right.$

Remark 5.3. Although from (3.3), $\delta_{\frac{\partial v_{\varepsilon}}{\partial x_{N}} /\left|\frac{\partial v_{\varepsilon}}{\partial x_{N}}\right|(x)} \otimes \varepsilon \mathbb{1}_{B_{\varepsilon}}\left|\frac{\partial v_{\varepsilon}}{\partial x_{N}}\right|^{2} d x$ possesses weak cluster points in the sense of the weak convergence $\stackrel{*}{\rightarrow}$ made precise above, for technical reason (proof of the sufficient conditions in Proposition 3.5 in [3]), it was not possible to state such a characterization for these cluster points because of possible concentration effects on the boundary of $\Sigma$. This is the reason why we deal with gradient Young-concentration measures localized on $\Sigma^{\prime} \subset \subset \Sigma$.

Taking into account that the 2-homogeneous extension $\tilde{\varphi}: \mathbb{R} \rightarrow \mathbb{R}$ of $\varphi \in \mathcal{C}\left(\mathbb{S}^{0}\right)$ satisfying $\varphi^{* *}>-\infty$ is of the form

$$
\varphi(\zeta)=\left\{\begin{array}{l}
c \zeta^{2} \text { if } \zeta \geq 0 \\
d \zeta^{2} \text { if } \zeta \leq 0
\end{array}\right.
$$

with $(c, d) \in \mathbb{R}^{+} \times \mathbb{R}^{+}$, the above characterization theorem can be reduced to the following (cf Corollary 3.6 in $[3])$

Corollary 5.4. A measure $\left(v, \mu_{\Sigma^{\prime}}=\left(a(x) \delta_{1}+b(x) \delta_{-1}\right) \otimes \pi\right)$ belongs to $\mathcal{Y C}\left(\Sigma^{\prime}\right)$ if and only if $v \in$ $W_{\Gamma_{0}}^{1,2}(\Omega \backslash \Sigma), \pi$ is concentrated on $\bar{\Sigma}^{\prime}$ and

$$
\frac{d \pi}{d \mathcal{H}^{N-1}\left\lfloor\Sigma^{\prime}\right.}(x)(a(x) c+b(x) d) \geq \varphi([v](x)) \text { for } \mathcal{H}^{N-1}\left\lfloor\Sigma^{\prime} \text { a.e. } x \text { and for all }(c, d) \in \mathbb{R}^{+} \times \mathbb{R}^{+}\right.
$$


where $\varphi(\zeta)=\left\{\begin{array}{ll}c \zeta^{2} & \text { if } \zeta \geq 0 \\ d \zeta^{2} & \text { if } \zeta \leq 0\end{array}\right.$.

As stated in [3] Remark 2.5, every sequence $\left(u_{\varepsilon}\right)_{\varepsilon>0}$ satisfying (3.3) generates a gradient Youngconcentration measure. Therefore every sequence $\left(\bar{u}_{\varepsilon}\right)_{\varepsilon>0}, \bar{u}_{\varepsilon} \in \operatorname{argmin} F_{\varepsilon}$, generates a measure $\bar{\mu}_{\Sigma^{\prime}} \in$ $\mathcal{Y C}\left(\Sigma^{\prime}\right)$. Let $\bar{u}$ be a strong limit of $\left(\bar{u}_{\varepsilon}\right)_{\varepsilon>0}$ in $L^{2}(\Omega)$, then, under the condition $g^{\infty, 2}\left(\hat{\xi}, \xi_{3}\right) \geq g^{\infty, 2}\left(\hat{0}, \xi_{3}\right)$, the next theorem states that the internal term $H_{i n}(\bar{\theta})(\operatorname{cf}(3.1))$ where $\bar{\theta}$ is the solution of $\inf _{\theta \in X(\bar{u})} H(\theta)$, possesses an integral representation with respect to the Young-concentration measure $\bar{\mu}_{\Sigma^{\prime}}$. In some sense we localize $H_{\text {in }}$ on $S \times\{ \pm 1\}$. Moreover, by using Theorem 5.2 we will deduce some bounds on $\bar{\mu}_{\Sigma^{\prime}}$.

Theorem 5.5. Let $\bar{u}_{\varepsilon}$ be a minimizer of $\min \left\{F_{\varepsilon}(v): v \in L^{2}(\Omega)\right\}$ and, for every $\Sigma^{\prime} \subset \subset \Sigma$, $\left(\bar{u}, \bar{\mu}_{\Sigma^{\prime}}\right)$ be a gradient Young-concentration measure localized on $\Sigma^{\prime}$ generated by the sequence $\left(\bar{u}_{\varepsilon}\right)_{\varepsilon>0}$. Then the two following assertions hold:

i) $\bar{u}_{\varepsilon} \rightarrow \bar{u}$ in $L^{2}(\Omega), F_{\varepsilon}\left(\bar{u}_{\varepsilon}\right) \rightarrow F_{0}(\bar{u})=\min \left\{F_{0}(u): u \in L^{2}(\Omega)\right\}$;

ii) Let $\mathcal{F}$ be a countable familly of $\Sigma^{\prime} \subset \subset \Sigma$, then there exists $\bar{\mu} \in \mathbb{M}\left(\bar{\Omega} \times \mathbb{S}^{0}\right), \bar{\mu}=\bar{\mu}_{\hat{x}} \otimes \bar{\pi}$ with $\bar{\pi}$ concentrated on $\bar{\Sigma}$ such that for all $\Sigma^{\prime} \in \mathcal{F}, \bar{\mu} \mid \bar{\Sigma}^{\prime} \times \mathbb{S}^{0}=\bar{\mu}_{\Sigma^{\prime}}$. Assume furthermore that $g^{\infty, 2}$ satisfies the condition

$$
\forall \xi \in \mathbb{R}^{3}, g^{\infty, 2}\left(\hat{\xi}, \xi_{3}\right) \geq g^{\infty, 2}\left(\hat{0}, \xi_{3}\right) .
$$

Then, every weak cluster point $\bar{\theta}$ of the sequence $\left(\tau_{\varepsilon} \bar{u}_{\varepsilon}\right)_{\varepsilon>0}$ in $V(B)$ satisfies $H(\bar{\theta})=\inf _{\theta \in X(\bar{u})} H(\theta)$ and

$$
\begin{aligned}
& \int_{-\frac{1}{2}}^{\frac{1}{2}} g^{\infty, 2}\left(\hat{0}, \frac{\partial \bar{\theta}}{\partial x_{N}}\right)(\hat{x}, s) d s=\frac{d \bar{\pi}}{d \hat{x}}(\hat{x}) \int_{\mathbb{S}^{0}} g^{\infty, 2}\left(\hat{0}, \xi_{3}\right) d \bar{\mu}_{\hat{x}} \text { for a.e. } \hat{x} \text { in } \Sigma^{\prime} ; \\
& H_{\text {in }}(\bar{\theta})=\int_{\Sigma}\left[\frac{d \bar{\pi}}{d \hat{x}}(\hat{x}) \int_{\mathbb{S}^{0}} g^{\infty, 2}\left(\hat{0}, \xi_{3}\right) d \bar{\mu}_{\hat{x}}\right] d \hat{x} .
\end{aligned}
$$

Proof. According to the variational nature of the $\Gamma$-convergence, for a subsequence one has

$$
\begin{aligned}
\bar{u}_{\varepsilon} \rightarrow \bar{u} \text { in } L^{2}(\Omega) & \\
\lim _{\varepsilon \rightarrow 0} F_{\varepsilon}\left(\bar{u}_{\varepsilon}\right) & =F_{0}(\bar{u})=\min \left\{F_{0}(v): v \in L^{2}(\Omega)\right\} \\
& =\int_{\Omega} f(\nabla \bar{u}) d x+\inf _{\theta \in X(\bar{u})} H(\theta) .
\end{aligned}
$$

Fix $\Sigma^{\prime} \subset \subset \Sigma$. From (3.3), for the subsequence (possibly dependent on $\Sigma^{\prime}$ ) associated with the gradient Young-concentration measure $\left(\bar{u}, \bar{\mu}_{\Sigma^{\prime}}\right)$, there exist a subsequence and a measure $\bar{\mu}=\bar{\mu}_{\hat{x}} \otimes \bar{\pi}$ in $\mathbb{M}\left(\bar{\Omega} \times \mathbb{S}^{0}\right)$ with $\bar{\pi}$ concentrated in $\bar{\Sigma}$, such that

$$
\delta_{\frac{\partial \bar{u}_{\varepsilon}}{\partial x_{N}} /\left|\frac{\partial \bar{u}_{\varepsilon}}{\partial x_{N}}\right|(x)} \otimes \varepsilon \mathbb{1}_{B_{\varepsilon}}\left|\frac{\partial \bar{u}_{\varepsilon}}{\partial x_{N}}\right|^{2} d x \rightarrow \bar{\mu} .
$$

Thus, from (3.11) and (5.2) we infer

$$
\begin{aligned}
\lim _{\varepsilon \rightarrow 0} \varepsilon^{2} \int_{B} g\left(\widehat{\nabla} \tau_{\varepsilon} \bar{u}_{\varepsilon}, \frac{1}{\varepsilon} \frac{\partial \tau_{\varepsilon} \bar{u}_{\varepsilon}}{\partial x_{N}}\right) d x & =\lim _{\varepsilon \rightarrow 0} \int_{B} g^{\infty, 2}\left(\varepsilon \widehat{\nabla} \tau_{\varepsilon} \bar{u}_{\varepsilon}, \frac{\partial \tau_{\varepsilon} \bar{u}_{\varepsilon}}{\partial x_{N}}\right) d x \\
& \geq \lim _{\varepsilon \rightarrow 0} \int_{B_{\varepsilon}} g^{\infty, 2}\left(\hat{0}, \frac{\partial \bar{u}_{\varepsilon}}{\partial x_{N}}\right) d x \\
& =\int_{\bar{\Sigma}}\left(\int_{\mathbb{S}^{0}} g^{\infty, 2}\left(\hat{0}, \xi_{3}\right) d \bar{\mu}_{\hat{x}}\right) d \bar{\pi}
\end{aligned}
$$

Let $\bar{\theta}$ be the weak limit of $\tau_{\varepsilon} \bar{u}_{\varepsilon}$ in $V(B)$ for the considered subsequence. Then, from (5.5) and since

$$
\liminf _{\varepsilon \rightarrow 0} \int_{\Omega_{\varepsilon}} f\left(\nabla \bar{u}_{\varepsilon}\right) d x \geq \int_{\Omega} f(\nabla \bar{u}) d x \text { and } \lim _{\varepsilon \rightarrow 0}\left\langle\mathcal{S}_{\varepsilon}, \tau_{\varepsilon} \bar{u}_{\varepsilon}\right\rangle=\langle\mathcal{S}, \bar{\theta}\rangle
$$


we infer

$$
\lim _{\varepsilon \rightarrow 0} F_{\varepsilon}\left(\bar{u}_{\varepsilon}\right) \geq \int_{\Omega} f(\nabla \bar{u}) d x+\int_{\bar{\Sigma}}\left(\int_{\mathbb{S}^{0}} g^{\infty, 2}\left(\hat{0}, \xi_{3}\right) d \bar{\mu}_{\hat{x}}\right) d \bar{\pi}-\langle\mathcal{S}, \bar{\theta}\rangle .
$$

Collecting (5.4) and (5.6) we obtain

$$
\int_{\Omega} f(\nabla \bar{u}) d x+\inf _{\theta \in X(\bar{u})} H(\theta) \geq \int_{\Omega} f(\nabla \bar{u}) d x+\int_{\bar{\Sigma}}\left(\int_{\mathbb{S}^{0}} g^{\infty, 2}\left(\hat{0}, \xi_{3}\right) d \bar{\mu}_{\hat{x}}\right) d \bar{\pi}-\langle\mathcal{S}, \bar{\theta}\rangle,
$$

in particular

$$
\int_{\Omega} f(\nabla \bar{u}) d x+H(\bar{\theta}) \geq \int_{\Omega} f(\nabla \bar{u}) d x+\int_{\bar{\Sigma}}\left(\int_{\mathbb{S}^{0}} g^{\infty, 2}\left(\hat{0}, \xi_{3}\right) d \bar{\mu}_{\hat{x}}\right) d \bar{\pi}-\langle\mathcal{S}, \bar{\theta}\rangle,
$$

thus

$$
\begin{aligned}
\int_{B} g^{\infty, 2}\left(\hat{0}, \frac{\partial \bar{\theta}}{\partial x_{N}}\right) d x & \geq \int_{\bar{\Sigma}}\left(\int_{\mathbb{S}^{0}} g^{\infty, 2}\left(\hat{0}, \xi_{3}\right) d \bar{\mu}_{\hat{x}}\right) d \bar{\pi} \\
& \geq \int_{\bar{\Sigma}} \frac{d \bar{\pi}}{d \hat{x}}(\hat{x})\left(\int_{\mathbb{S}^{0}} g^{\infty, 2}\left(\hat{0}, \xi_{3}\right) d \bar{\mu}_{\hat{x}}\right) d \hat{x} .
\end{aligned}
$$

On the other hand, by a standard lower semicontinuity argument, for every $\varphi \in \mathcal{C}_{c}(\Sigma), \varphi \geq 0$,

$$
\begin{aligned}
\liminf _{\varepsilon \rightarrow 0} \int_{B} \varphi(\hat{x}) g^{\infty, 2}\left(\hat{0}, \frac{\partial \tau_{\varepsilon} \bar{u}_{\varepsilon}}{\partial x_{N}}\right) d x & =\int_{\Sigma} \varphi(\hat{x})\left(\int_{\mathbb{S}^{0}} g^{\infty, 2}\left(\hat{0}, \xi_{3}\right) d \bar{\mu}_{\hat{x}}\right) d \bar{\pi} \\
& \geq \int_{B} \varphi(\hat{x}) g^{\infty, 2}\left(\hat{0}, \frac{\partial \bar{\theta}}{\partial x_{N}}\right) d x
\end{aligned}
$$

so that

$$
\int_{-\frac{1}{2}}^{\frac{1}{2}} g^{\infty, 2}\left(\hat{0}, \frac{\partial \bar{\theta}}{\partial x_{N}}\right)(\hat{x}, s) d s \leq \frac{d \bar{\pi}}{d \hat{x}}(\hat{x}) \int_{\mathbb{S}^{0}} g^{\infty, 2}\left(\hat{0}, \xi_{3}\right) d \bar{\mu}_{\hat{x}} \quad \text { for a.e. } \hat{x} \in \Sigma .
$$

Combining (5.7) and (5.8) we deduce

$$
\int_{-\frac{1}{2}}^{\frac{1}{2}} g^{\infty, 2}\left(\hat{0}, \frac{\partial \bar{\theta}}{\partial x_{N}}\right)(\hat{x}, s) d s=\frac{d \bar{\pi}}{d \hat{x}}(\hat{x}) \int_{\mathbb{S}^{0}} g^{\infty, 2}\left(\hat{0}, \xi_{3}\right) d \bar{\mu}_{\hat{x}} \quad \text { for a.e. } \hat{x} \in \Sigma .
$$

Clearly, $\bar{\mu} \mid \bar{\Sigma}^{\prime} \times \mathbb{S}^{0}=\bar{\mu}_{\Sigma^{\prime}}$. Now, by using a standard Cantor's diagonal process, the same equality holds for all $\Sigma^{\prime}$ of the countable familly $\mathcal{F}$. It remains to show that $H(\bar{\theta})=\inf _{\theta \in X(\bar{u})} H(\theta)$. It's enough to notice that

$$
\begin{aligned}
\lim _{\varepsilon \rightarrow 0} F_{\varepsilon}\left(\bar{u}_{\varepsilon}\right) & =\int_{\Omega} f(\nabla \bar{u}) d x+\inf _{\theta \in X(\bar{u})} H(\theta) \\
& \geq \liminf _{\varepsilon \rightarrow 0} \int_{\Omega_{\varepsilon}} f\left(\nabla \bar{u}_{\varepsilon}\right) d x+\liminf _{\varepsilon \rightarrow 0}\left(\varepsilon^{2} \int_{B} g\left(\widehat{\nabla} \tau_{\varepsilon} \bar{u}_{\varepsilon}, \frac{1}{\varepsilon} \frac{\partial \tau_{\varepsilon} \bar{u}_{\varepsilon}}{\partial x_{N}}\right) d x-\left\langle\mathcal{S}_{\varepsilon}, \tau_{\varepsilon} \bar{u}_{\varepsilon}\right\rangle\right) \\
& \geq \int_{\Omega} f(\nabla \bar{u}) d x+\int_{B} g^{\infty, 2}\left(\hat{0}, \frac{\partial \bar{\theta}}{\partial x_{N}}\right) d x-\langle\mathcal{S}, \bar{\theta}\rangle \\
& =\int_{\Omega} f(\nabla \bar{u}) d x+H(\bar{\theta})
\end{aligned}
$$

which completes the proof.

We define the following two constants associated with the function $g$ :

$$
c(g):=\min \left(\frac{g^{\infty, 2}(\widehat{0},-1)}{g^{\infty, 2}(\widehat{0}, 1)}, \frac{g^{\infty, 2}(\widehat{0}, 1)}{g^{\infty, 2}(\widehat{0},-1)}\right), C(g)=\frac{1}{c(g)}=\max \left(\frac{g^{\infty, 2}(\widehat{0},-1)}{g^{\infty, 2}(\widehat{0}, 1)}, \frac{g^{\infty, 2}(\widehat{0}, 1)}{g^{\infty, 2}(\widehat{0},-1)}\right) .
$$


Recall that

$$
g^{\infty, 2}(\widehat{0}, \xi)=\left\{\begin{array}{cll}
g^{\infty, 2}(\widehat{0},-1)|\xi|^{2} & \text { if } & \xi \leq 0 \\
g^{\infty, 2}(\widehat{0}, 1)|\xi|^{2} & \text { if } & \xi>0
\end{array} .\right.
$$

Moreover, from the assumption on the function $g$, clearly,

$$
g^{\infty, 2}(\widehat{0}, 1)>0 \text { and } g^{\infty, 2}(\widehat{0},-1)>0 .
$$

We make precise the probability measure $\bar{\mu}_{\hat{x}}$ localized on $\Sigma^{\prime} \subset \subset \Sigma$ as follows:

$$
\bar{\mu}_{\hat{x}}:=p(\hat{x}) \delta_{1}+q(\hat{x}) \delta_{-1} \quad \text { with } \quad p(\hat{x})+q(\hat{x})=1 \text { a.e. } \hat{x} \in \Sigma^{\prime} .
$$

Corollary 5.6. Under the assumptions of Theorem 5.5, the three following estimates hold:

(i) for a.e. $\hat{x}$ in $\Sigma^{\prime}$

$$
c(g) \int_{-\frac{1}{2}}^{\frac{1}{2}}\left|\frac{\partial \bar{\theta}}{\partial x_{N}}(\hat{x}, s)\right|^{2} d s \leq \frac{d \bar{\pi}}{d x_{N}}(\hat{x}) \leq C(g) \int_{-\frac{1}{2}}^{\frac{1}{2}}\left|\frac{\partial \bar{\theta}}{\partial x_{N}}(\hat{x}, s)\right|^{2} d s,
$$

$$
\text { and } \frac{d \bar{\pi}}{d x_{N}}(\hat{x})=\int_{-\frac{1}{2}}^{\frac{1}{2}}\left|\frac{\partial \bar{\theta}}{\partial x_{N}}(\hat{x}, s)\right|^{2} d s \quad \text { when } g^{\infty, 2}(\widehat{0},-1)=g^{\infty, 2}(\widehat{0}, 1) \text {; }
$$

(ii) $\frac{c(g)|[\bar{u}](\hat{x})|^{2}}{\int_{-\frac{1}{2}}^{\frac{1}{2}}\left|\frac{\partial \bar{\theta}}{\partial x_{N}}(\hat{x}, s)\right|^{2} d s} \leq p(\hat{x}) \leq 1$ for a.e. $\hat{x}$ such that $[\bar{u}](\hat{x})>0$;

(iii) $\frac{c(g)|[\bar{u}](\hat{x})|^{2}}{\int_{-\frac{1}{2}}^{\frac{1}{2}}\left|\frac{\partial \bar{\theta}}{\partial x_{N}}(\hat{x}, s)\right|^{2} d s} \leq q(\hat{x}) \leq 1$ for a.e. $\hat{x}$ such that $[\bar{u}](\hat{x})<0$.

Proof. Since $\bar{\mu}_{\widehat{x}}=p(\widehat{x}) \delta_{1}+q(\widehat{x}) \delta_{-1}$, we have $\int_{\mathbb{S}^{0}} g^{\infty, 2}(\xi) d \bar{\mu}_{\widehat{x}}=p(\widehat{x}) g^{\infty, 2}(\widehat{0}, 1)+q(\widehat{x}) g^{\infty, 2}(\widehat{0},-1)$ with $p(\widehat{x})+q(\widehat{x})=1$ a.e. $\widehat{x}$ in $\Sigma^{\prime}$ so that from (5.3), one has

$$
\begin{aligned}
\int_{-\frac{1}{2}}^{\frac{1}{2}} g^{\infty, 2}\left(\widehat{0}, \frac{\partial \bar{\theta}}{\partial x_{N}}(\widehat{x}, s)\right) d s & =\left(\int_{\mathbb{S}^{0}} g^{\infty, 2}(\xi) d \bar{\mu}_{\hat{x}}\right) \frac{d \bar{\pi}}{d \widehat{x}}(\widehat{x}) \\
& =\frac{d \bar{\pi}}{d \widehat{x}}(\widehat{x})\left\{p(\widehat{x}) g^{\infty, 2}(\widehat{0}, 1)+q(\widehat{x}) g^{\infty, 2}(\widehat{0},-1)\right\} \text { a.e. } \widehat{x} \in \Sigma^{\prime}
\end{aligned}
$$

We are going to establish

$$
c(g) \int_{-\frac{1}{2}}^{\frac{1}{2}}\left|\frac{\partial \bar{\theta}}{\partial x_{N}}(\widehat{x}, s)\right|^{2} d s \leq \frac{d \bar{\pi}}{d \widehat{x}}(\widehat{x}) \leq C(g) \int_{-\frac{1}{2}}^{\frac{1}{2}}\left|\frac{\partial \bar{\theta}}{\partial x_{N}}(\widehat{x}, s)\right|^{2} d s .
$$

From (5.10) we deduce that

$$
\begin{aligned}
\min \left\{g^{\infty, 2}(\widehat{0},-1), g^{\infty, 2}(\widehat{0}, 1)\right\} \int_{-\frac{1}{2}}^{\frac{1}{2}}\left|\frac{\partial \bar{\theta}}{\partial x_{N}}(\widehat{x}, s)\right|^{2} d s & \leq \int_{-\frac{1}{2}}^{\frac{1}{2}} g^{\infty, 2}\left(\widehat{0}, \frac{\partial \bar{\theta}}{\partial x_{N}}(\widehat{x}, s)\right) d s \\
& =\left(\int_{\mathbb{S}^{0}} g^{\infty, 2}(\xi) d \bar{\mu}_{x}\right) \frac{d \bar{\pi}}{d \widehat{x}}(\widehat{x}) \\
& =\left\{p(\widehat{x}) g^{\infty, 2}(\widehat{0}, 1)+q(\widehat{x}) g^{\infty, 2}(\widehat{0},-1)\right\} \frac{d \bar{\pi}}{d \widehat{x}}(\widehat{x}) \\
& \leq \max \left\{g^{\infty, 2}(\widehat{0},-1), g^{\infty, 2}(\widehat{0}, 1)\right\} \frac{d \bar{\pi}}{d \widehat{x}}(\widehat{x})
\end{aligned}
$$


and

$$
\begin{aligned}
\min \left\{g^{\infty, 2}(\widehat{0},-1), g^{\infty, 2}(\widehat{0}, 1)\right\} \frac{d \bar{\pi}}{d \widehat{x}}(\widehat{x}) & =\min \left\{g^{\infty, 2}(\widehat{0},-1), g^{\infty, 2}(\widehat{0}, 1)\right\}\{p(\widehat{x})+q(\widehat{x})\} \frac{d \bar{\pi}}{d \widehat{x}}(\widehat{x}) \\
& \leq\left\{p(\widehat{x}) g^{\infty, 2}(\widehat{0}, 1)+q(\widehat{x}) g^{\infty, 2}(\widehat{0},-1)\right\} \frac{d \bar{\pi}}{d \widehat{x}}(\widehat{x}) \\
& =\left(\int_{\mathbb{S}^{0}} g^{\infty, 2}(\xi) d \bar{\mu}_{x}\right) \frac{d \bar{\pi}}{d \widehat{x}}(\widehat{x}) \\
& =\int_{-\frac{1}{2}}^{\frac{1}{2}} g^{\infty, 2}\left(\widehat{0}, \frac{\partial \bar{\theta}}{\partial x_{N}}(\widehat{x}, s)\right) d s \\
& \leq \max \left\{g^{\infty, 2}(\widehat{0},-1), g^{\infty, 2}(\widehat{0}, 1)\right\} \int_{-\frac{1}{2}}^{\frac{1}{2}}\left|\frac{\partial \bar{\theta}}{\partial x_{N}}(\widehat{x}, s)\right|^{2} d s(5
\end{aligned}
$$

Then, from (5.11) and (5.12) we have

$$
c(g) \int_{\frac{-1}{2}}^{\frac{1}{2}}\left|\frac{\partial \bar{\theta}}{\partial x_{N}}(\widehat{x}, s)\right|^{2} d s=\frac{\min \left\{g^{\infty, 2}(\widehat{0},-1), g^{\infty, 2}(\widehat{0}, 1)\right\}}{\max \left\{g^{\infty, 2}(\widehat{0},-1), g^{\infty, 2}(\widehat{0}, 1)\right\}} \int_{-\frac{1}{2}}^{\frac{1}{2}}\left|\frac{\partial \bar{\theta}}{\partial x_{N}}(\widehat{x}, s)\right|^{2} d s \leq \frac{d \bar{\pi}}{d \widehat{x}}(\widehat{x})
$$

and

$$
\frac{d \bar{\pi}}{d \widehat{x}}(\widehat{x}) \leq \frac{\max \left\{g^{\infty, 2}(\widehat{0},-1), g^{\infty, 2}(\widehat{0}, 1)\right\}}{\min \left\{g^{\infty, 2}(\widehat{0},-1), g^{\infty, 2}(\widehat{0}, 1)\right\}} \int_{-\frac{1}{2}}^{\frac{1}{2}}\left|\frac{\partial \bar{\theta}}{\partial x_{N}}(\widehat{x}, s)\right|^{2} d s=C(g) \int_{\frac{-1}{2}}^{\frac{1}{2}}\left|\frac{\partial \bar{\theta}}{\partial x_{N}}(\widehat{x}, s)\right|^{2} d s
$$

from which we deduce,

$$
c(g) \int_{-\frac{1}{2}}^{\frac{1}{2}}\left|\frac{\partial \bar{\theta}}{\partial x_{N}}(\widehat{x}, s)\right|^{2} d s \leq \frac{d \bar{\pi}}{d \widehat{x}}(\widehat{x}) \leq C(g) \int_{-\frac{1}{2}}^{\frac{1}{2}}\left|\frac{\partial \bar{\theta}}{\partial x_{N}}(\widehat{x}, s)\right|^{2} d s .
$$

Let us prove (ii) and (iii). According to Theorem 5.2, for every $\varphi \in \mathcal{C}\left(\mathbb{S}^{0}\right)$ such that $\varphi^{* *}>-\infty$,

$$
\frac{d \bar{\pi}}{d \mathcal{H}_{\left[\Sigma^{\prime}\right.}^{N-1}}(x) \int_{\mathbb{S}^{0}} \varphi(\zeta) d \bar{\mu}_{x} \geq \varphi^{* *}([v](x)) \text { for } \mathcal{H}^{N-1} \text { a.e. } x \in \Sigma^{\prime},
$$

where $\bar{\pi}=\frac{d \bar{\pi}}{d \mathcal{H}_{\left[\Sigma^{\prime}\right.}^{N-1}} \mathcal{H}_{\left[\Sigma^{\prime}\right.}^{N-1}+\bar{\pi}_{s}$ is the Radon-Nikodym decomposition of $\bar{\pi}$ with respect to the measure $\mathcal{H}_{\left[\Sigma^{\prime}\right.}^{N-1}$. We assume that $[\bar{u}](\hat{x})>0$ and show that

$$
\frac{c(g)|[\bar{u}](\hat{x})|^{2}}{\int_{-\frac{1}{2}}^{\frac{1}{2}}\left|\frac{\partial \bar{\theta}}{\partial x_{N}}(\hat{x}, s)\right|^{2} d s} \leq p(\hat{x}) \leq 1
$$

Let

$$
\varphi(\xi)=\left\{\begin{array}{cll}
\varphi(1)|\xi|^{2} & \text { if } & \xi \geq 0 \\
0 & \text { if } & \xi<0
\end{array} .\right.
$$

Clearly, $\varphi^{* *}([\bar{u}](\widehat{x}))=\varphi([\bar{u}](\widehat{x}))=\varphi(1)|[u](\widehat{x})|^{2}$. From the inequality (5.13), it follows that

$$
\begin{aligned}
\varphi(1)|[\bar{u}](\widehat{x})|^{2} & =\varphi^{* *}([\bar{u}](\widehat{x})) \\
& \leq \frac{d \bar{\pi}}{d \widehat{x}}(\widehat{x})\left(\int_{\mathbb{S}^{0}} \varphi(\xi) d \bar{\mu}_{x}\right) \\
& \leq C(g)\left(\int_{-\frac{1}{2}}^{\frac{1}{2}}\left|\frac{\partial \bar{\theta}}{\partial x_{N}}(\widehat{x}, s)\right|^{2} d s\right)\left(\int_{\mathbb{S}^{0}} \varphi(\xi) d \bar{\mu}_{x}\right) \\
& =C(g) p(\widehat{x}) \varphi(1) \int_{-\frac{1}{2}}^{\frac{1}{2}}\left|\frac{\partial \bar{\theta}}{\partial x_{N}}(\widehat{x}, s)\right|^{2} d s .
\end{aligned}
$$


Then, we obtain

$$
\frac{|[\bar{u}](\hat{x})|^{2}}{C(g) \int_{-\frac{1}{2}}^{\frac{1}{2}}\left|\frac{\partial \bar{\theta}}{\partial x_{N}}(\hat{x}, s)\right|^{2} d s}=\frac{c(g)|[\bar{u}](\hat{x})|^{2}}{\int_{-\frac{1}{2}}^{\frac{1}{2}}\left|\frac{\partial \bar{\theta}}{\partial x_{N}}(\hat{x}, s)\right|^{2} d s} \leq p(\hat{x}) \leq 1 .
$$

The proof of (iii) is similar.

\section{References}

1. H. Attouch. Variational Convergence for Functions and Operators. Applicable Mathematics Series. Pitman Advanced Publishing Program, 1985.

2. D. Caillerie. The Effect of a Thin Inclusion of High Rigidity in an Elastic Body. Math. Meth. in the Appl. Sci, 2 (1980), 251-270.

3. G. Croce, C. Lacour, G. Michaille. A characterization of gradient Young-concentration measures generated by solutions of Dirichlet-type problems with large sources ESAIM Control Optim. Calc. Var. 15 (2009), no. 4, 818-838.

4. E. De Giorgi. Sulla convergenza di alcune successioni d'integrali del tipo dell' aera Rend. Matematica, (1975), no. 8, 277-294.

5. H. Federer. Geometric Measure Theory. Classic in Mathematics, Springer-Verlag 1969.

6. I. Fonseca, S. Müller, P. Pedregal. Analysis of concentration and oscillation effects generated by gradients. Siam J. Math. Anal. 29 (1998), no 3, 736-756.

7. G. Geymonat, F. Krasucki, S. Lenci. Mathematical analysis of a bounded joint with a soft thin adhesive. Mathematics and Mechanics of Solids, 4 (1999), 201-225.

8. D. Kinderlehrer, P. Pedregal. Characterization of Young measures generated by gradients. Arch. Rational Mech. Anal. 119 (1991), 329-365.

9. C. Licht, G. Michaille. A modelling of elastic adhesive bonded joints. Advances in Mathematical Sciences and Applications, 7 (1997), no 2, 711-740.

10. C. Licht, G. Michaille, S. Pagano. A model of elastic adhesive bonded joints through oscillationconcentration measures. J. Math. Pures Appl. (9) 87 (2007), no. 4, 343-365. 